\title{
Analytical model for predicting tool wear in orthogonal machining of unidirectional carbon fibre reinforced polymer (CFRP)
}

\author{
Lukas Seeholzer $^{1}$ (D) $\cdot$ Thomas Krammer $^{2} \cdot$ Parvaneh Saeedi $^{2} \cdot$ Konrad Wegener $^{1}$
}

Received: 12 July 2021 / Accepted: 29 October 2021 / Published online: 24 January 2022

(c) The Author(s) 2022

\begin{abstract}
Progressive tool wear due to abrasive carbon fibres is one of the main issues in machining of CFRP and responsible for the short tool life. Because of occurring wear during machining, the tool's micro-geometry changes continuously resulting in higher process forces and an increasing risk for workpiece damages. In this paper, a novel analytical model is presented in order to predict the wear-related change of the micro-geometry in orthogonal machining of CFRP depending on the fibre orientation and the initial tool geometry. For this purpose, a concept called the wear rate distribution is introduced which represents a measure to quantify the wear rate along the active micro-geometry. Based on experimental investigation, it is shown that the shape of an arbitrary wear rate distribution between two closely spaced wear states can be approximated and parameterised with a "line - curve - line" approach. Using the authors' previously published analytical force model, the wear rate distribution can be calculated as function of five wear parameters that are used to parameterise the active micro-geometry of an arbitrary wear state. Based on an iterative solver, this is used to simulate the tool wear progression during machining. For model validation, the simulation is compared to experimental data in terms of the cutting edge profiles, the amount of worn tool material and the process forces. Accordingly, the wear model is capable to reproduce the most important wear characteristics, e.g. the cutting edge rounding, the decreasing clearance angle and the increasing contact length at the flank face.
\end{abstract}

Keywords Fibre-reinforced plastic $\cdot$ Cutting operation $\cdot$ Wear $\cdot$ Modelling

\section{Introduction}

As exemplarily stated by Davim [1], carbon fibre reinforced polymer (CFRP) is characterised by high specific strength and stiffness properties, a high corrosion resistance and a low thermal expansion behaviour. Based on the associated high potential for lightweight construction, CFRP has found increasing application as a high-performance engineering material in commercial aerospace and high-end automotive industries [2, 3]. According to Geier and Pereszlai [4], CFRP components are usually designed and manufactured nearnet-shape; however, conventional machining operations are usually required for machining difficult-to-mould features,

Lukas Seeholzer

seeholzer@iwf.mavt.ethz.ch

1 Institute of Machine Tools and Manufacturing (IWF), ETH Zürich, Leonhardstrasse 21, 8092 Zurich, Switzerland

2 School of Engineering Science, Simon Fraser University, 8888 University Dr, Burnaby, BC V5A 1S6, Canada increasing the surface quality or fulfilling other dimensional requirements.

Analytical force models can be used to estimate the machining forces based on process-, tool- and materialspecific influencing parameters. By showing causalities, e.g. the influence of varying tool angles or the feed rate on the thrust force, analytical force models represent an important tool for gaining process insights that subsequently can be used for efficient tool or process optimisation [5-7]. Accordingly, they help to reduce the tool development time and the number of experimental test series. During the last decades, numerous researchers have focussed on developing analytical force models for orthogonal machining of unidirectional (UD) CFRP material. This is attributed to the reduced complexity of the process-specific machining kinematics and the associated possibility of a differentiated experimental process analysis $[8,9]$. As stated by Wan et al. [10], the gained process understanding in terms of modelling the fundamental machining mechanics can be used for developing force models for more complex machining operations, e.g. 
drilling and milling processes. In the following, a brief literature review about former analytical force modelling approaches in orthogonal machining of UD-CFRP material is presented.

In 1995, Bhatnagar et al. [11] published one of the first analytical force models that allows the estimation of process forces in machining fibre cutting angles in the range of $0^{\circ} \leq \Phi \leq 180^{\circ}$. In this context, the fibre cutting angle $\Phi$ is defined as the angle between the cutting velocity direction and the fibre orientation. Similar to the force model presented by Takeyama and Iijima [12] for glass fibre reinforced polymer (GFRP), Bhatnagar et al. [11] used the Merchant's shear-plane model [13] for the analytical prediction of process forces. For this purpose, Bhatnagar et al. [11] assumed the shear plane angle to be identical to the fibre cutting angle while axial tension was used as the fibre fracture criterion. In 2001, Zhang et al. [14] presented a mechanical force model, where the contact zone of the cutting edge was separated into three different regions represented by the rake and clearance faces and the cutting edge rounding. The authors assumed that the total cutting and thrust forces can be determined as superpositions of the corresponding force components in the three regions that are derived based on fundamental concepts of elastic contact mechanics. Although neither complex fibre/matrix-interactions nor component-specific failure mechanisms were considered, this early force model was able to show basic causalities, e.g. the influences of the rake angle, the fibre orientation and the depth of cut on the cutting and feed forces. In the following years, different subsequent modelling approaches $[8,15,16]$ adopted the basic idea of Zhang et al. [14] in terms of the three-part contact region, but used a more detailed multiphase configuration of the CFRP material in order to consider component-specific deformations and failure mechanisms. In 2010, Jahromi and Bahr [16] proposed a force model which uses a representative volume element (RVE) concept to predict the process forces for fibre cutting angles in the range of $90^{\circ} \leq \Phi \leq 180^{\circ}$. The authors used the bending deformation of the RVE and the required energy via the minimum potential energy principle (MPEP) to link the cutting motion of the tool to the resulting machining forces. Qi et al. [15] followed a similar approach and presented an analytical force model for fibre cutting angles in the range of $0^{\circ} \leq \Phi \leq 90^{\circ}$. Subsequently, $\mathrm{Xu}$ and Zhang [17, 18] presented a mechanical force model that allows the prediction of cutting and thrust forces not only for conventional but also vibration-assisted machining. While Jahromi and Bahr [16] and Qi et al. [15] conditioned occurring fibre fracture in their models on the second derivative of the bent RVE, Xu and Zhang [17, 18] considered the three-dimensional stress state to calculate an equivalent tensile stress in the contact region which then was compared to the fibre's tensile strength. In order to calculate the threedimensional stresses in the contact region, $\mathrm{Xu}$ and Zhang
$[17,18]$ simplified the complex contact situation between the carbon fibre and the tool with the elastic contact between two cylinders.

As stated by Wang et al. [19], machining of CFRP is associated with excessive mechanical wear due to the frictional contact between the tool surface and the highly abrasive carbon fibres during the machining operation. Based on an experimental study, Seeholzer et al. [20] showed that the wear-related material loss at the cutting tool results in a continuously changing tool shape and is predominated by an enlargement of the cutting edge rounding and a decreasing clearance angle. As the tool's micro-geometry changes due to wear, this means that the contact situation between the cutting tool and the machined CFRP material varies as well [8]. Representative for several experimental studies [21-24], Henerichs et al. [25] revealed that the cutting and thrust forces increase significantly during the machining operation because of progressive tool wear and the abovementioned wear effects along the tool's contact region. Furthermore, the authors showed that the wear-related change of the tool's micro-geometry and its effect on process forces strongly depend on the initial tool geometry and the fibre cutting angle. According to Henerichs et al. [25], the most intense tool wear appears in machining of CFRP material with a fibre cutting angle in the range of $30^{\circ} \leq \Phi \leq 90^{\circ}$, while for $150^{\circ} \leq \Phi \leq 0$, only minor wear changes were identified. Furthermore, the authors stated that occurring flank wear can be reduced clearly by increasing the initial clearance angle.

As concluded by Wan et al. [10], most existing analytical force models do not consider tool wear for the prediction of process forces. As a direct consequence, the wearrelated increase of process forces mentioned before cannot be simulated with these modelling approaches. According to Ramulu [26], the risk for process-related damages at the workpiece, e.g. delamination, splintering, fibre pull-outs and cracking, increases for higher process forces and thus becomes more important when the tool wear progresses. Consequently, tool wear has to be taken into account for the development process of cutting tools. As a conclusion, there is a need for a sufficiently detailed force model, which in cooperation with an appropriate wear model enables the prediction of the tool wear progression and the corresponding machining forces. A brief literature review about former analytical models that consider tool wear effects for force prediction is presented in the following.

In 2018, Voss et al. [8] proposed an analytical force model that allows the approximation of the cutting and thrust forces during orthogonal machining of UD-CFRP material as functions of the fibre cutting angle, the initial shape of the tool geometry and its wear-related change with increasing cutting length. For this purpose, the authors introduced five wear parameters $\gamma^{*}, \alpha^{*}, l_{\gamma}, l_{\alpha}$ and $b_{c}$ that are used to parameterise the general shape of a worn 
cutting edge geometry using a "line - ellipse - line" approach. Based on orthogonal machining experiments, these five wear parameters were evaluated for different wear states at different cutting lengths and then are used as input data for the analytical force model via look-up tables. According to the performed model validation, the simulated cutting and thrust forces as well as their wear-related increases with the cutting length were in good agreement to the experiments. Zhang et al. [27] presented a theoretical model for predicting the thrust forces in drilling and countersinking of CFRP. For this purpose, the authors separated the contact area of the cutting edge into four deformation regions, namely the rake face, the cutting edge rounding and the flank face with and without elastic spring back. For each deformation region, representative force components were calculated based on previously published modelling concepts [14, 28, 29]. In order to consider tool wear effects for force modelling, the flank wear land along the cutting edge was determined experimentally and quantified as piecewise function for each considered wear state. Subsequently, these piecewise functions were used as additional inputs for the force model. During the model validation in terms of the thrust force, maximum errors of $7.18 \%$ for drilling and $7.12 \%$ for countersinking were determined. Bai et al. [30] presented a force model for predicting the thrust force in drilling of UD-CFRP material under consideration of tool wear effects. Similar to the approach of Voss et al. [8], the authors divided the contact region of the cutting edge into three regions and used an ellipse with its minor and major semi-axes to approximate the shape of the cutting edge rounding. In order to consider tool wear effects in the modelling approach, the minor and major semi-axes were evaluated experimentally and then used as additional inputs for the force model, comparable to the approaches of Voss et al. [8] and Zhang et al. [27]. Assuming orthogonal cutting conditions for an infinitesimal element of the cutting edge, representative force components were calculated for each of the three regions and then integrated along the main cutting edges. For calculating the force components of the chisel edge, the force model of Guo et al. [29] was applied. The common deficiency of the aforementioned analytical force models is the fact that the prediction of process forces is only possible if the shape of the worn cutting tool is already known since no wear models are included. A first approach of a coupled force and wear simulation is presented by Luo et al. [31] in 2018. The authors presented a mechanistic model for predicting the thrust force in drilling CFRP/titanium-stacks for a new as well as a worn tool geometry. For this purpose, the authors used the cutting edge radius as a quantitative indicator of tool wear and applied Archard's wear model in order to formulate a mathematical relation between the cutting edge radius, the contact force, the cutting length and the wedge angle. Based on a performed model validation, the authors showed that the simulation agrees well with the experimental data.

In this paper, a novel wear modelling approach is presented, which in cooperation with the previously published analytical force model [8] allows the prediction of progressive tool wear in machining of UD-CFRP material depending on the fibre cutting angle and the initial tool geometry. The tool wear prediction covers the simulation of the cutting edge's wear-related change, the amount of removed tool material along the contact area and the corresponding cutting and thrust forces as functions of the cutting length or the machining time. As a result, look-up tables are no longer required for the wear parameters $\gamma^{*}, \alpha^{*}, l_{\gamma}$ and $l_{\alpha}$ in the analytical force model [8] as they are calculated anew during the wear simulation. In the scope of a comprehensive model validation, the applicability and the achievable prediction accuracy of the proposed wear simulation are analysed for different combinations of the fibre cutting angle and the initial rake and clearance angles of the cutting tool. The simulated results are generally in good agreement with the experimental data and the wear model is capable to reproduce the most important wear characteristics. The nomenclature used in the paper is summarised in Table 1.

\section{Fundamentals in modelling tool wear in orthogonal machining of CFRP}

\subsection{Influence of tool wear on the tool's contact situation}

According to Fig. 1, only a small region of the cutting tool is in contact with the workpiece material during the orthogonal machining operation. This operative part of the cutting edge is defined as the tool's active micro-geometry and bounded by the nominal material level ahead of the cutting edge represented by the contact point $A$ and the last tool/workpiece contact point $D$ on the flank face. Because of the elastic spring back phenomenon, typically found in machining of CFRP, the point $D$ on the flank face is different to the tool's foremost point $C$ in feed direction. The distance between the points $C$ and $D$ in feed direction is defined as the bouncing back height $b_{c}$ and represents a measure for the elastic spring back of the CFRP material [8]. The contact point $B$ represents the foremost point in cutting velocity direction. By definition, mechanical wear only occurs along the contact region between the points $A$ and $D$. In this work, the fibre cutting angle $\Phi$ is defined as the angle between the cutting velocity direction and the fibre orientation measured counter-clockwise as shown in Fig. 1 b. 
Table 1 Nomenclature

\begin{tabular}{|c|c|c|c|c|c|}
\hline$A, B, C, D$ & $\begin{array}{l}\text { Contact points defining the active micro- } \\
\text { geometry }\end{array}$ & $(-)$ & $r_{f}$ & Radius of the carbon fibre & $(\mu \mathrm{m})$ \\
\hline$C_{1}, C_{2}$ & $\begin{array}{l}\text { Fitting parameters according to Usui's } \\
\text { wear rate model }\end{array}$ & $(-)$ & $r_{\text {peak }}$ & Cutting edge radius & $(\mu \mathrm{m})$ \\
\hline$A_{1}, A_{2}, A_{3}$ & $\begin{array}{l}\text { Fitting parameters for the presented wear } \\
\text { rate model }\end{array}$ & $(-)$ & $R 1, R 2, R 3$ & Abbreviations for the regions 1,2 and 3 & $(-)$ \\
\hline$A_{W}$ & Cross section of removed tool material & $\left(\mu \mathrm{m}^{2}\right)$ & $T_{\text {cut }}$ & Cutting temperature & $\left({ }^{\circ} \mathrm{C}\right)$ \\
\hline$A_{W_{-} i}(i=1,2,3)$ & $\begin{array}{l}\text { Region-specific cross section of removed } \\
\text { tool material }\end{array}$ & $\left(\mu \mathrm{m}^{2}\right)$ & $t_{c}$ & $\begin{array}{l}\text { Distance between the points } \mathrm{C}_{\mathrm{i}} \text { and } \mathrm{C}_{\mathrm{i}+1} \\
\text { in feed direction }\end{array}$ & $(\mu \mathrm{m})$ \\
\hline$a_{c}$ & $\begin{array}{l}\text { Actual cutting depth in orthogonal } \\
\text { machining }\end{array}$ & $(\mu \mathrm{m})$ & $\begin{array}{l}v_{S_{i} i} \\
(i=1,2,3)\end{array}$ & Sliding velocity in R1, R2 and R3 & $(\mathrm{m} / \mathrm{min})$ \\
\hline$a_{e}$ & $\begin{array}{l}\text { Machining width }=\text { workpiece width } \\
\text { (full section) }\end{array}$ & $(\mathrm{mm})$ & $\begin{array}{l}\bar{v}_{S_{-} i} \\
(i \stackrel{-1}{=} 1,3)\end{array}$ & Mean sliding velocity in $\mathrm{R} 1, \mathrm{R} 2$ and $\mathrm{R} 3$ & $(\mathrm{~m} / \mathrm{min})$ \\
\hline$b$ & Wall thickness of the CFRP workpiece & $(\mathrm{mm})$ & $\mathrm{W}_{\mathrm{i}}$ and $\mathrm{W}_{\mathrm{i}+1}$ & $\begin{array}{l}\text { Active micro-geometry for two consecu- } \\
\text { tive wear states }\end{array}$ & $(-)$ \\
\hline$b_{c}$ & $\begin{array}{l}\text { Bouncing back height of the CFRP } \\
\text { material }\end{array}$ & $(\mu \mathrm{m})$ & $W_{\max }$ & Maximum of the wear rate distribution & $(-)$ \\
\hline$f$ & Feed rate & $(\mathrm{mm})$ & 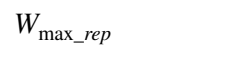 & $\begin{array}{l}\text { Representative maximum of the wear } \\
\text { rate distribution }\end{array}$ & $(-)$ \\
\hline$F_{c i}(i=1,2,3)$ & Region-specific cutting force & $(\mathrm{N})$ & $\bar{w}$ & Mean wear rate & $\left(\mu \mathrm{m}^{2} / \mathrm{m}\right)$ \\
\hline $\bar{F}_{k_{i} i}(i=1,2,3)$ & Region-specific contact force & $(\mathrm{N})$ & $\bar{w}_{i}(i=1,2,3)$ & Mean wear rate in R1, R2 and R3 & $\left(\mu \mathrm{m}^{2} / \mathrm{m}\right)$ \\
\hline$F_{t_{-} i}(i=1,2,3)$ & Region-specific thrust force & $(\mathrm{N})$ & $z_{r_{-} i}(i=A, B, C, D)$ & $\begin{array}{l}\text { Magnitude of the wear rate distributions } \\
\text { at the points } \mathrm{A}, \mathrm{B}, \mathrm{C} \text { and } \mathrm{D}\end{array}$ & $(\mu \mathrm{m} / \mathrm{m})$ \\
\hline$l_{\text {cut }}$ & Cutting length & (m) & $z_{r_{-} W \max }$ & $\begin{array}{l}\text { Magnitude of the wear rate distribution } \\
\text { at the point } \mathrm{W}_{\max }\end{array}$ & $(\mu \mathrm{m} / \mathrm{m})$ \\
\hline$l_{\text {cut_int }}$ & $\begin{array}{l}\text { Cutting length increment for interpola- } \\
\text { tion }\end{array}$ & $(\mathrm{m})$ & $v_{i j}$ & Wear vector at the point $\mathrm{P}_{\mathrm{ij}}$ & $(-)$ \\
\hline$l_{r}$ & Arc length & $(\mu \mathrm{m})$ & $v_{c}$ & Cutting velocity & $(\mathrm{m} / \mathrm{min})$ \\
\hline$l_{r_{i} i}(i=1,2,3)$ & Arc length of $\mathrm{R} 1, \mathrm{R} 2$ and $\mathrm{R} 3$ & $(\mu \mathrm{m})$ & $v_{f}$ & Feed velocity & $(\mathrm{m} / \mathrm{min})$ \\
\hline$l_{r_{i} i}(i=A, B, C, D)$ & Arc length of the points A, B, C, D & $(\mu \mathrm{m})$ & $\alpha, \gamma$ & Initial clearance and rake angles & $\left({ }^{\circ}\right)$ \\
\hline$l_{r_{-} W \max }$ & Arc length of the point $\mathrm{W}_{\max }$ & $(\mu \mathrm{m})$ & $\alpha^{*}$ & $\begin{array}{l}\text { Wear parameter; Clearance angle in the } \\
\text { tool's worn state }\end{array}$ & $\left({ }^{\circ}\right)$ \\
\hline$l_{r_{-} 2 \_l e f t}$ & $\begin{array}{l}\text { Horizontal distance between the points } \\
\mathrm{B} \text { and } \mathrm{W}_{\max } \text { in the wear rate distribu- } \\
\text { tion }\end{array}$ & $(\mu \mathrm{m})$ & $\gamma^{*}$ & $\begin{array}{l}\text { Wear parameter; Rake angle in the tool's } \\
\text { worn state }\end{array}$ & $\left({ }^{\circ}\right)$ \\
\hline$l_{3}$ & $\begin{array}{l}\text { Distance between the points } \mathrm{C} \text { and } \mathrm{D} \text { in } \\
\text { cutting velocity direction }\end{array}$ & $(\mu \mathrm{m})$ & $\Delta l_{\text {cut }}$ & Cutting length increment for simulation & (m) \\
\hline$l_{\alpha}$ & $\begin{array}{l}\text { Wear parameter; Distance between the } \\
\text { points } \mathrm{C} \text { and } \mathrm{B} \text { in cutting velocity } \\
\text { direction }\end{array}$ & $(\mu \mathrm{m})$ & $\sigma_{k \_i}(i=1,2,3)$ & Contact stress in R1, R2 and R3 & (MPa) \\
\hline$l_{\gamma}$ & $\begin{array}{l}\text { Wear parameter; Distance between the } \\
\text { points } \mathrm{C} \text { and } \mathrm{B} \text { in feed direction }\end{array}$ & $(\mu \mathrm{m})$ & $\bar{\sigma}_{k \_i}(i=1,2,3)$ & Mean contact stress in R1, R2 and R3 & (MPa) \\
\hline $\begin{array}{l}n_{w_{-} i} \\
\left(i=0^{\circ}, 30^{\circ}, 60^{\circ}, 90^{\circ}\right)\end{array}$ & Ratio between $l_{r_{-} 2 \_l e f t}$ and $l_{r_{-} 2}$ & $(-)$ & $\Phi$ & Fibre cutting angle & $\left({ }^{\circ}\right)$ \\
\hline
\end{tabular}

In Fig. 1b, the dotted red line describes the active microgeometry of an exemplary wear state $W_{i}$ of the cutting edge with the corresponding contact points $A_{i}, B_{i}, C_{i}$ and $D_{i}$. Beginning from the wear state $W_{i}$, the resumption of the machining operation will result in a series of reactions that are explained in the following. Once the cutting edge is in contact with the material, the abrasive carbon fibres rub against the tool surface of the active micro-geometry $W_{i}$ and therefore cause material loss due to mechanical wear. As experimentally shown by different researchers [25, 33, 34], the tool's material loss along $W_{i}$ is usually the most intense close to the tool tip and decreases in directions of $A_{i}$ and $D_{i}$. This is explained by the irregular distributed tool load along the contact zone resulting in a variable wear potential and hence material loss. An irregular distribution of material loss along the contact region means that the resulting tool shape after continuing the machining operation is different to that of $W_{i}$. According to Sheikh-Ahmad [34], the 


\section{Real machining operation}

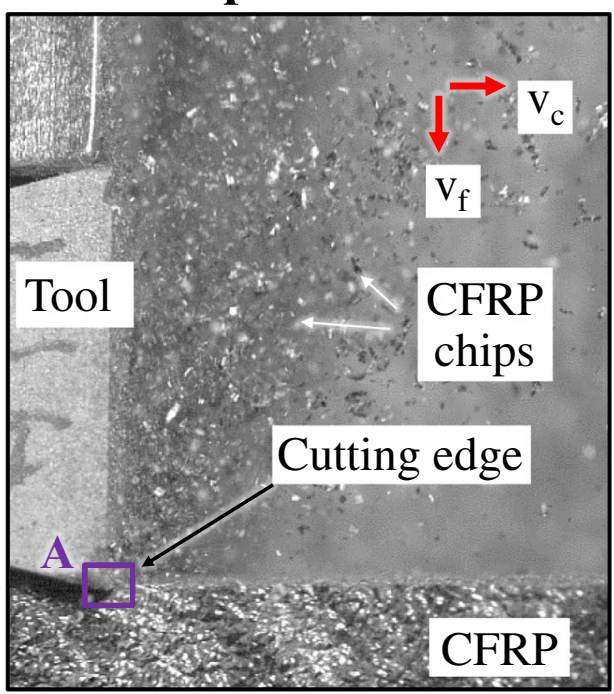

(a)
Actual contact area A defined as the active micro-geometry

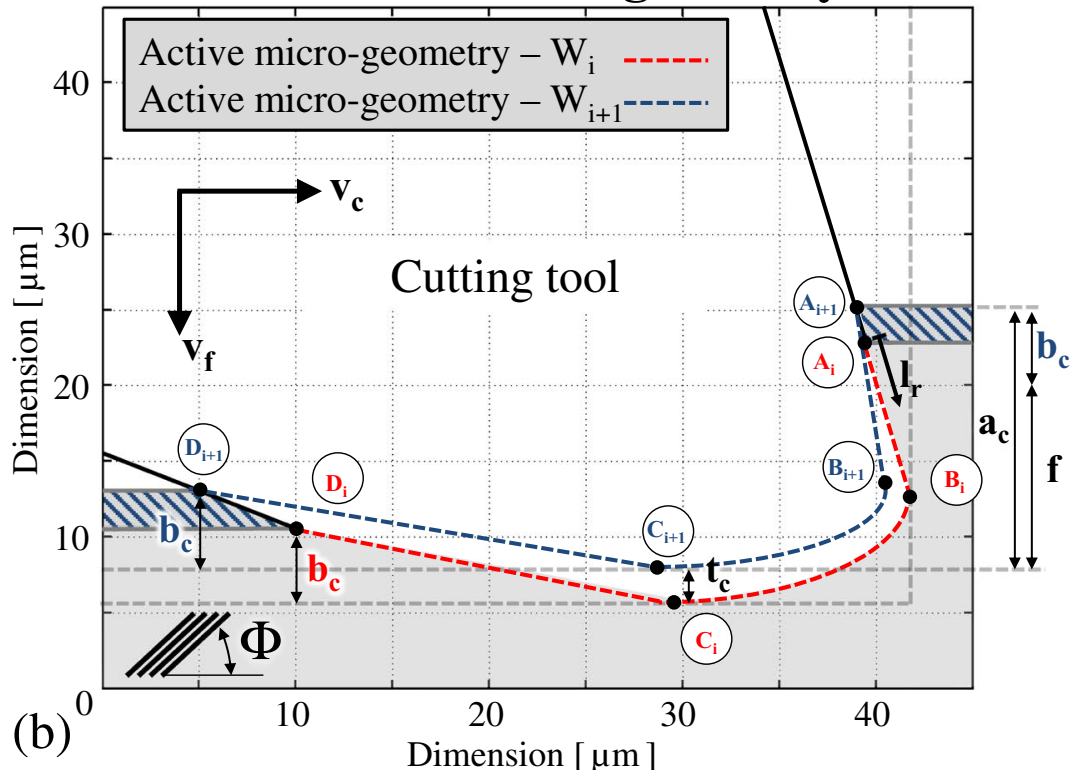

Fig. 1 (a) Snap shot of a real orthogonal machining situation with UD-CFRP material, (b) schematic illustration of the tool's contact region by means of the active micro-geometry for two consecutive wear states denoted by $W_{i}$ and $W_{i+1}$ [32]

corresponding change in tool/workpiece contact has a direct influence on the machining process in terms of the process forces and the level of elastic spring back. In this context, independent experimental studies revealed that the process forces [21-24] and the intensity of the elastic spring back in terms of the bouncing back height $[8,35]$ increase as tool wear progresses.

In an orthogonal machining process, where the CFRP material is removed layer by layer, the machined surface is reprocessed in each new cutting edge crossing. Therefore, an increasing bouncing back height means that the actual depth of cut $a_{c}$, which corresponds to the distance between the points $C$ and $A$ in feed direction, changes during the machining operation. Assuming that the difference in spring back between two consecutive spindle rotations is negligible, the value of $a_{c}$ can be formulated as a function of $b_{c}$, the programmed feed $f$ and the parameter $t_{c}$, describing the distance between the points $C_{i}$ and $C_{i+1}$ in feed direction.

$a_{c}=f+b_{c}-t_{c}$

As the value of $b_{c}$ increases much faster than $t_{c}$, the actual depth of cut increases as tool wear progresses, which means that previously untouched rake and flank face material of the cutting tool gradually gets in contact with the CFRP $[8,36]$. This is illustrated in Fig. 1b, where the dotted blue line represents the updated active micro-geometry $W_{i+1}$ after continuing the machining operation for a short period.
The CFRP material which is in contact with the previously untouched rake and flank faces is indicated with the blue/ grey striped area. The enclosed area between the contours of $W_{i}$ and $W_{i+l}$ corresponds to the cross-section of the removed tool material $A_{W}$.

\subsection{Shape quantification of the active micro-geometry}

According to Voss et al. [8], the shape of an arbitrary active micro-geometry $W_{i}$ can be approximated with a "line - ellipse line" approach using the five wear parameters $\gamma^{*}, \alpha^{*}, l_{\gamma}, l_{\alpha}$ and $b_{c}$. As this shape parameterisation strategy is applied in this work, it is briefly explained in the following while for detailed information, it is referred to the original publication. Figure 2a shows the active micro-geometries for a new and a worn cutting edge. For their force model, Voss et al. [8] followed the example of Zhang et al. [14] and separated the tool's active micro-geometry into three regions. These regions are the rake face, the cutting edge rounding and the flank face that in the following are abbreviated with R1, R2 and R3. As stated by Voss et al. [8], R1 and R3 retain their nearly straight shape also with progressive tool wear, but the corresponding rake and clearance angles change from $\gamma$ to $\gamma^{*}$ and $\alpha$ to $\alpha^{*}$ due to irregular material loss. In the tool's new state, R2 has a circular shape with a predefined peak radius $r_{\text {peak }}$. However, as documented by numerous 
New and worn cutting edge (measured)

Parameterised cutting edge

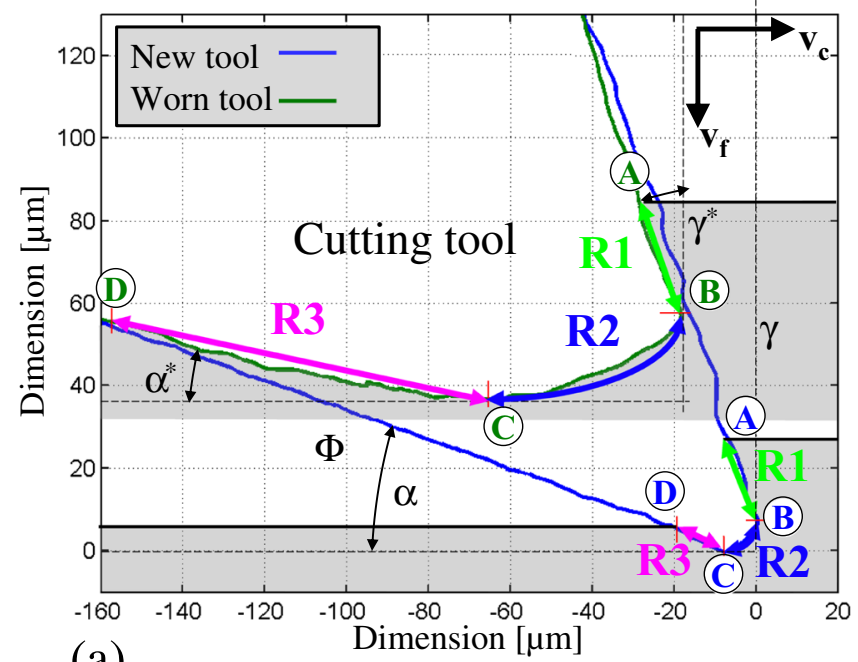

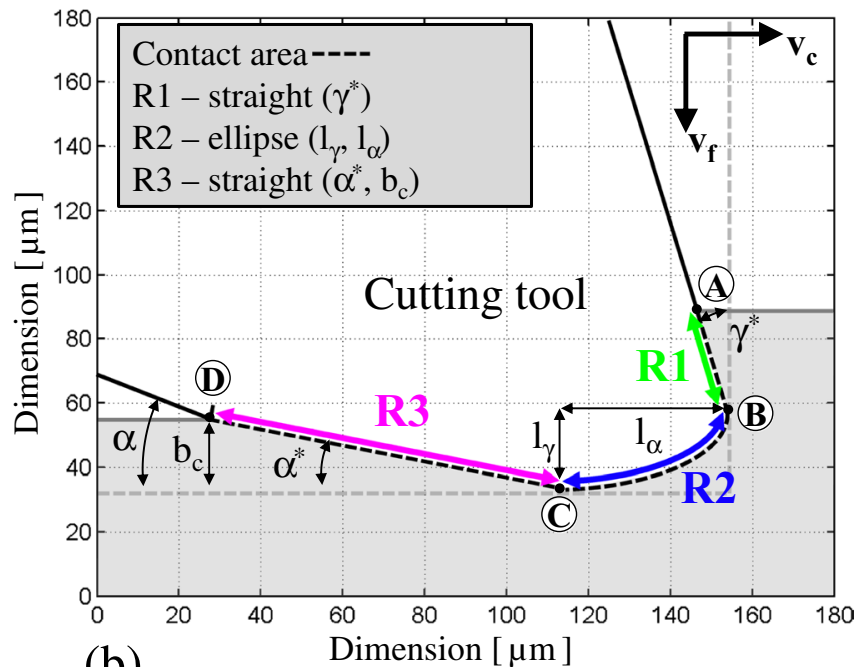

(b)

Fig. 2 (a) Exemplary new and worn cutting edge profile with R1, R2 and R3, and (b) shape parametrisation of the active micro-geometry using the five wear parameters $\gamma^{*}, \alpha^{*}, l_{\gamma}, l_{\alpha}$ and $b_{c}$ according to Voss et al. [8]

experimental studies [21-25], progressive tool wear in R2 causes an increasing asymmetric cutting edge rounding with more material loss on the flank face side. Notwithstanding that, Voss et al. [8] showed that the shape of R2 for an arbitrary wear state can be well approximated with the major and minor semi-axes $l_{\alpha}$ and $l_{\gamma}$ of an ellipse. The fifth wear parameter is the bouncing back height $b_{c}$ which is already introduced in Sect. 2.1. Figure $2 \mathrm{~b}$ shows the approximated shape of a representative active microgeometry using the five wear parameters $\gamma^{*}, \alpha^{*}, l_{\gamma}, l_{\alpha}$ and $b_{c}$.

\subsection{Distribution of tool wear and its quantification}

Assuming that the cutting tool does not fracture nor experience chipping during the machining operation, the wear process can be considered continuous. Therefore, the initial cutting edge profile smoothly morphs into the subsequent profiles as tool wear progresses. While the shape of the active micro-geometry changes due to the irregular material loss described in Sect. 2.1, the contact length between the points $A$ and $D$ increases because of the higher intensity of the elastic spring back.

In order to quantify the contact length between the points $A$ and $D$ for a given active micro-geometry $W_{i}$, the arc length $l_{r}$ is defined. According to Fig. 1b, the arc length $l_{r}$ starts at point $A$ and follows the actual shape of the active micro-geometry in direction of the points $B, C$ and $D$. In this work, the parameters $l_{r_{-} A}, l_{r_{-} B}, l_{r_{-} C}$ and $l_{r_{-} D}$ describe the individual arc lengths for the points $A, B, C$ and $D$. Since the starting point of the arc length is defined to be point $A$, the parameter $l_{r_{-} A}$ is always identical to zero. In contrast, the remaining parameters $l_{r_{-} B}, l_{r_{-} C}$ and $l_{r_{-} D}$ depend on the active micro-geometry and hence change as tool wear progresses. In general, the wear rate is defined as a material loss per unit of time. As discussed in Sect. 2.1, the amount of removed tool material changes along the contact area of the active micro-geometry, which means that the value of the wear rate depends on the considered contact length. In this context, it can be distinguished between a mean wear rate $\mathrm{w}_{\text {mean }}$ and a point-specific wear rate $w_{P S}$. The mean wear rate neglects the non-uniform material loss along the active-micro-geometry and is defined as the area loss per unit contact length per unit of time.

$\mathrm{w}_{\text {mean }}=\frac{A_{W}}{l_{r} t}$

In Eq. (2), the parameter $t$ represents the machining duration between $W_{i}$ and $W_{i+1}$. Since the non-uniform material removal is not considered, the value of $\mathrm{w}_{\text {mean }}$ does not provide information about how the tool wear is distributed along the active micro-geometry. If instead of $l_{r}$, only a small fraction of $l_{r}$ is used in Eq. (2); this results in a more accurate mean wear rate for the considered contact length increment. Accordingly, for an infinitesimal value of $l_{r}$, the calculated mean wear rate according to Eq. (2) equals to the pointspecific wear rate for one specific point of the cutting edge which is identical to $w_{P S}$. In order to allow the quantification of the wear rate along the active micro-geometry and thus as a function of the arc length, a concept called "wear vectors" is introduced. For this purpose, a set of points $P_{i j}$ is 


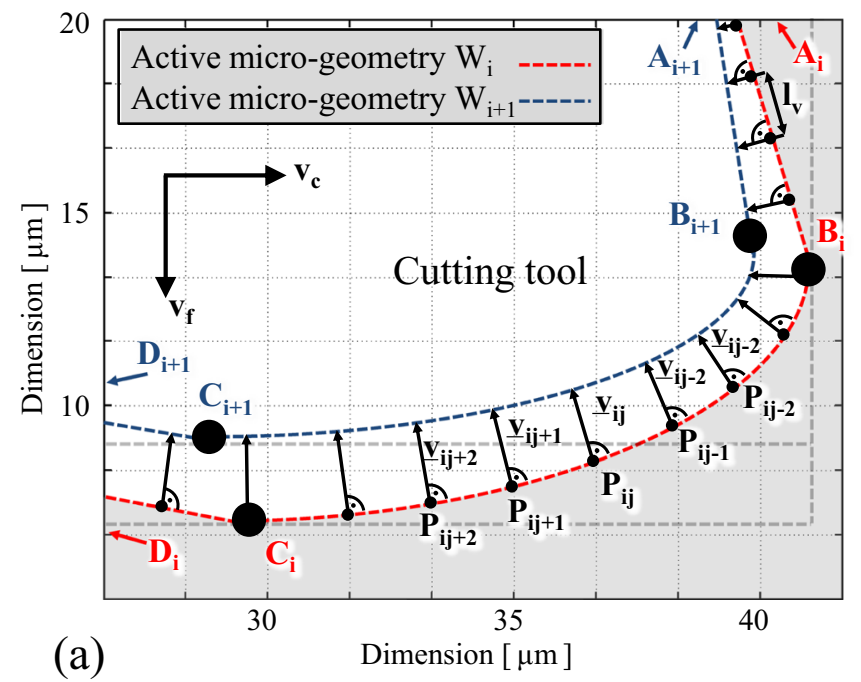

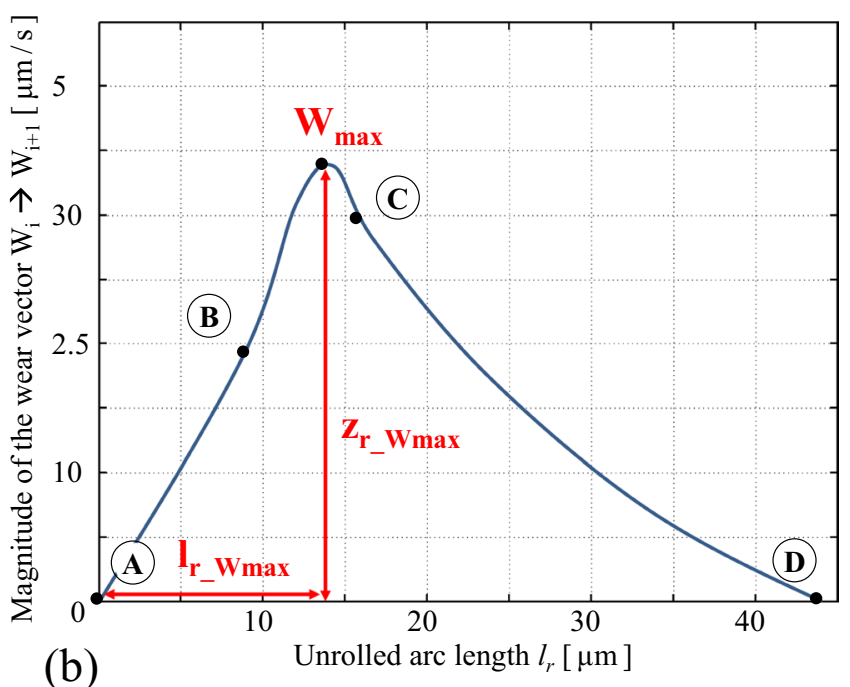

(b)

Fig. 3 (a) Enlarged view of R2 for the wear states $W_{i}$ and $W_{i+1}$ with drawn wear vectors, and (b) schematic illustration of the wear rate distribution (WRD) between $W_{i}$ and $W_{i+1}$ with its maximum represented by the point $W_{\max }$ and its coordinates $l_{r_{-} W m a x}$ and $z_{r_{-} \text {Wamx }}$

distributed along the arc length of the known active microgeometry $W_{i}$ via a predefined arc length interval $l_{v}$. This is shown in Fig. 3a using an enlarged point spacing due to visibility reasons. The first index " $i$ " of $P_{i j}$ refers to the corresponding wear state $W_{i}$. The second index " $j$ " refers to the specific point number with respect to the direction of counting in positive $l_{r}$-direction beginning in point $A$, see Fig. 3a. Subsequently, for each point $P_{i j}$ on $W_{i}$, the direction of the corresponding wear vector $\underline{v}_{i j}$ is defined to point in direction of $W_{i+1}$ and to be orthogonal to the shape of $W_{i}$. According to Fig. 3a, the magnitude of the wear vector $\left.\left|\underline{v}_{i j}\right|\right|^{i}$ is defined as the orthogonal distance from an arbitrary point $P_{i j}$ on $W_{i}$ to the next profile $W_{i+1}$.

The wear rate associated to a given wear vector can be represented by the wear vector's magnitude divided by the step size between $W_{i}$ and $W_{i+l}$ and can be interpreted as an orthogonal change in position of a point per unit of time. Consequently, it can be interpreted as a wear speed. Assuming the cutting velocity to be constant during the machining operation, the cutting distance is directly proportional to the machining time. As a result, the step size between $W_{i}$ and $W_{i+1}$ can be expressed as a difference in cutting length or machining time. Once the magnitudes of all wear vectors between $W_{i}$ and $W_{i+1}$ are known, the total amount of removed tool material between these profiles, that differ in machining time and cutting length, can be approximated by the sum of the products of wear vector magnitudes $\left|\underline{v}_{i j}\right|$ and the chosen arc length interval $l_{v}$. This approximation represents a sum of rectangular segments between $W_{i}$ and $W_{i+1}$ and is the more accurate the smaller the value of $l_{v}$.
The introduction of the wear vectors set the foundation of formulating a so-called wear rate distribution (WRD) which can be used as a measure to quantify the distribution of the wear rate along the arc length of an arbitrary active micro-geometry. The WRD is defined as the plotted magnitudes of the wear vectors with respect to their position along the active micro-geometry. An exemplary WRD is shown in Fig. 3b. The maximum of the WRD is defined to be $W_{\max }$ and can be described by its coordinates $l_{r_{-} W \max }$ and $z_{r_{-} W \max }$ representing the corresponding arc length and wear vectors magnitude for a predefined step size. Analogously, the parameters $z_{r_{-} A}, z_{r_{-} B}, z_{r_{-} C}$ and $z_{r_{-} D}$ represent the functional values of the WRD at the points $A, B, C$ and $D$. It has to be noted that the area under the curve of the WRD is not identical to $A_{W}$ but can be approximated by multiplying the wear vector's magnitudes with the corresponding arc length intervals $l_{v}$ as mentioned before. Depending on whether the step size between $W_{i}$ and $W_{i+1}$ is specified in metres cutting length or seconds machining time, the unit for the $y$-axis of the WRD in Fig. 3b is $\mu \mathrm{m} / \mathrm{m}$ or $\mu \mathrm{m} / \mathrm{s}$.

\section{Experimental investigation of the wear rate distribution}

With the concept of the WRD, an effective method is introduced in order to quantify the tool's wear rate between two arbitrary active micro geometries $W_{i}$ and $W_{i+l}$ as a function of $l_{r}$. This section deals with an experimental investigation of the WRD with the objective of analysing its characteristics in terms of its shape and size depending on the initial tool geometry, the fibre cutting angle and the cutting 
length. For this purpose, fundamental orthogonal machining experiments with UD-CFRP material were performed as preliminary work that is published in [20]. The goal of this extensive experimental study was the investigation of the wear-related change of the tool's active micro geometry with increasing cutting length for different combinations of the initial tool geometry and the fibre cutting angle. In this work, parts of these experimental results are used for investigating the characteristic of the WRD as well as for the final model validation.

\subsection{Fundamental orthogonal machining experiments with UD-CFRP material}

Seeholzer et al. [20] used a longitudinal face turning process installed on a CNC lathe type Okuma LB15-II in order to approximate the machining conditions of a non-interrupted orthogonal cut for a maximum cutting length of $l_{\text {cut }}=35 \mathrm{~m}$. For the experiments, the UD-CFRP material M21/34\%/ UD194/IMA-12 $k$ is used which contains $34 \%$ (by weight) epoxy-based matrix material type Hexply® $M 21$ and $66 \%$ intermediate modulus carbon fibre-type IMA- $12 \mathrm{k}$. This material is available with different fibre orientations which can be used to realise turning operations with different fibre cutting angles. In total, the authors performed orthogonal machining operations with eight different initial tool geometries and four different fibre cutting angles. Table 2 shows a list of all combinations of rake and clearance angles with the corresponding identification letters of the cutting inserts and the machined fibre cutting angles that are used in this work. Accordingly, these are the tool $\mathrm{H}(10 / 21)$ in machining $\Phi=0^{\circ}, \Phi=30^{\circ}, \Phi=60^{\circ}$ and $\Phi=90^{\circ}$ and the tools E (10/7), I (10/21) and L (20/14) in machining $\Phi=0^{\circ}$.

All experiments are performed with a cutting velocity of $v_{c}=90 \mathrm{~m} / \mathrm{min}$, a feed rate of $f=0.03 \mathrm{~mm} / \mathrm{rev}$ and a cutting width of $a_{e}=5 \mathrm{~mm}$. For each combination in Table 2, the orthogonal machining process is interrupted at five different cutting lengths and the actual wear state of the cutting

Table 2 List of all combinations of tool geometries and the machined fibre cutting angles that are used in this work

\begin{tabular}{lllll}
\hline Tool geometry & E & H & I & L \\
\hline Initial rake angle $\gamma\left({ }^{\circ}\right)$ & 10 & 10 & 10 & 20 \\
Initial clearance angle & 7 & 14 & 21 & 14 \\
$\quad \alpha\left(^{\circ}\right)$ & & & & \\
Abbreviation & E (10/7) & H (10/14) & I (10/21) & L (20/14) \\
Fibre cutting angle & & & & \\
$\Phi=0^{\circ}$ & $\checkmark$ & $\checkmark$ & $\checkmark$ & $\checkmark$ \\
$\Phi=30^{\circ}$ & & $\checkmark$ & & \\
$\Phi=60^{\circ}$ & & $\checkmark$ & & \\
$\Phi=90^{\circ}$ & & $\checkmark$ & & \\
\hline
\end{tabular}

insert is acquired as a point cloud using optical 3D microscopy. The considered cutting lengths are $l_{\text {cut }}=0 \mathrm{~m}, l_{\text {cut }}=5 \mathrm{~m}$, $l_{\text {cut }}=10 \mathrm{~m}, l_{\text {cut }}=15 \mathrm{~m}, l_{\text {cut }}=20 \mathrm{~m}$ and $l_{\text {cut }}=35 \mathrm{~m}$. For a precise evaluation of the tool's wear affected zone at each considered cutting length, the flank faces of the cutting inserts are previously marked by short pulsed laser processing. The laser-affected zone is only related to a very thin top-layer of the carbide tools and vanishes if it gets in contact with the bounced back CFRP material. As explained in detail in the original work [20], this allows the optical evaluation of the last tool/workpiece contact point on the flank face represented by point $D$. For the quantification of the worn tool profiles, the authors used Voss's "line - ellipse - line" parameterisation approach based on the five wear parameters $\gamma^{*}, \alpha^{*}, l_{\gamma}, l_{\alpha}$ and $b_{c}$ discussed in Sect. 2.2.

\subsection{Calculation of the wear rate distribution based on the experimental data in [20]}

Figure 4a shows an overlay of an exemplary series of measured cutting edge profiles with respect to the evaluated cutting lengths of $l_{\text {cut }}=0 \mathrm{~m}, l_{\text {cut }}=5 \mathrm{~m}, l_{\text {cut }}=10 \mathrm{~m}, l_{\text {cut }}=15 \mathrm{~m}$, $l_{\text {cut }}=20 \mathrm{~m}$ and $l_{\text {cut }}=35 \mathrm{~m}$. Following the terminology of this study, the colours green, blue and magenta are used for the three regions R1, R2 and R3 of the active micro-geometry. The black parts of the plotted cutting edge profiles represent the unworn tool areas that are not in contact with the CFRP material during the machining operation. Figure $4 \mathrm{~b}$ shows the measured values of the arc length $l_{r}$ and the worn tool area $A_{W}$ with respect to the analysing steps.

There are two major limitations when using the concept of wear vectors to evaluate the WRD between two arbitrary cutting edge profiles. The first limitation arises if the orthogonal trajectory from a point $P_{i j}$ on profile $W_{i}$ never intersects profile $W_{i+1}$. This is exemplary shown in Fig. 4a with the representative wear vector $\underline{v}_{1}$. The second limitation of using wear vectors is that by decreasing the arc length interval $l_{v}$, adjacent wear vectors are more likely to intersect each other before intersecting with the consecutive cutting edge profile $W_{i+1}$. Intersections of wear vectors have to be prevented since they would cause a distortion of the calculated WRD. As shown in Fig. 4 a with the representative wear vectors $\underline{v}_{2}$ and $\underline{v}_{3}$, an intersection of wear vectors occurs when the magnitude of a wear vector is greater than the radius of curvature at the respective point on $W_{i}$ and the arc length interval $l_{v}$ is sufficiently small.

Both limiting conditions of the wear vectors can be eliminated if the orthogonal distance from profile $W_{i}$ to $W_{i+1}$ is always less than the radius of curvature at every point along the profile $W_{i}$. One approach to reduce the orthogonal distance between two consecutive wear profiles is to take cutting edge measurements more often during the orthogonal machining process, which means that more measured cutting 
Overlay of measured cutting edge profiles

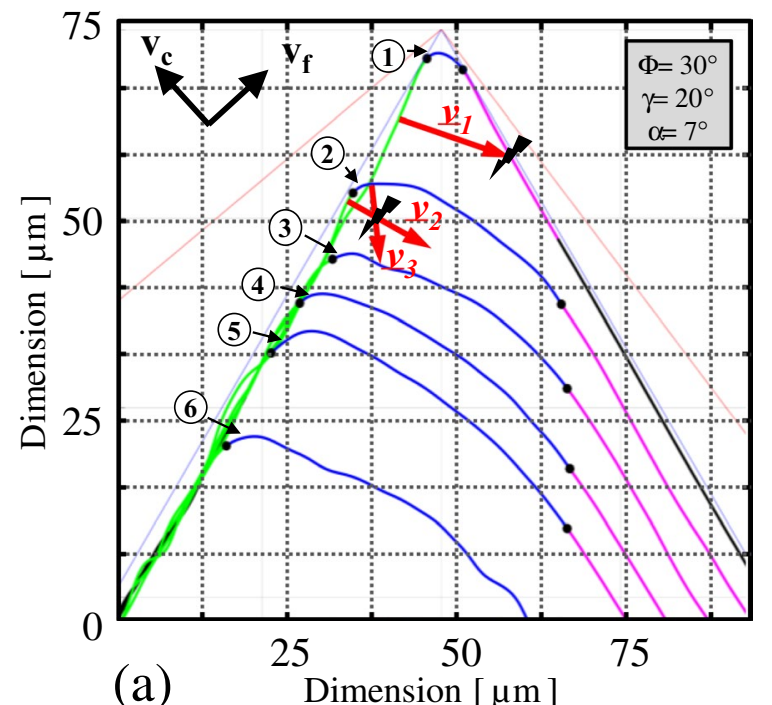

Measured values for $I_{r}$ and $A_{W}$

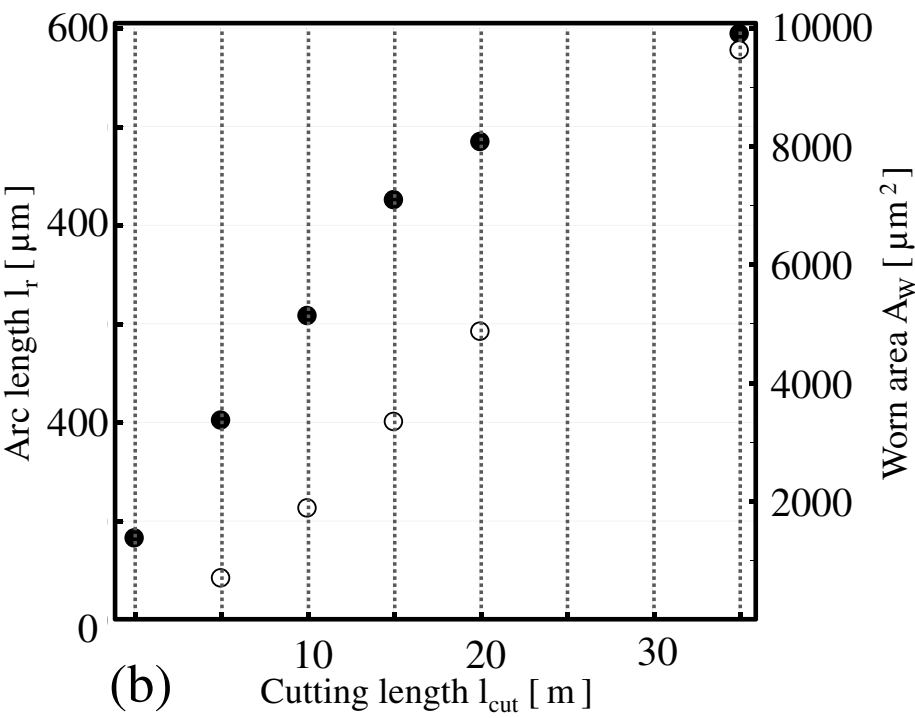

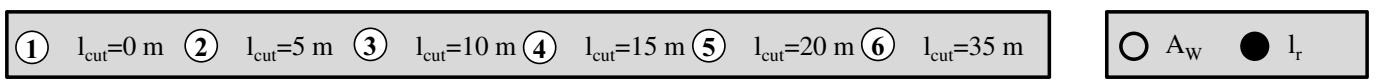

Fig. 4 (a) Exemplary overlay of measured cutting edge profiles including the visualisation of wear vectors limitations based on [20], and (b) measured values for the arc length $l_{r}$ and the worn area $A_{W}$ with respect to the evaluated cutting lengths

edge profiles with smaller differences in cutting length would be available. However, this approach is not practical, since this method would increase the error associated with interrupting the cutting process due to the run-in period when continuing the machining operation after analysing the micro-geometry. Another approach, which is applied in this study, is to use the already existing measured cutting edge profiles at $l_{\text {cut }}=0 \mathrm{~m}, l_{\text {cut }}=5 \mathrm{~m}, l_{\text {cut }}=10 \mathrm{~m}, l_{\text {cut }}=15 \mathrm{~m}$, $l_{\text {cut }}=20 \mathrm{~m}$ and $l_{\text {cut }}=35 \mathrm{~m}$ and to apply a reasonable interpolating strategy to generate additional wear profiles in between the experimental determined profiles. In the following Sect. 3.2.1, the applied interpolation strategy is explained in detail. Subsequently, Sect. 3.2.2 gives an overview of the determined WRD using the measured as well as the interpolated cutting edge profiles.

\subsubsection{Interpolating strategy to generate additional cutting edge profiles}

As a preparation for the applied interpolating method, the contact points $A, B, C$ and $D$ have to be localised for each measured cutting edge profile. For this purpose, first, the contact points $B$ and $C$, describing the foremost points in cutting and feed velocity directions, have to be localised. The contact point $D$ can be determined using the laser marks on the flank face of the cutting inserts according to Sect. 3.1. Subsequently, the information of $b_{c}$ and $t_{c}$ can be used to calculate the actual depth of cut $a_{c}$ according to Eq. (1). In accordance with the schematic illustration in Fig. 1, this can be used to localise the contact point $A$ on the rake face. Once the positions of the contact points $A, B, C$ and $D$ are known, the entire active micro-geometry for each measured wear state can be separated into the three regions R1, R2 and R3 as discussed in Sect. 2.2. For each measured active microgeometry, the region-specific arc lengths $l_{r_{-} 1}, l_{r_{-} 2}$ and $l_{r_{-} 3}$ can be calculated as follows:

$l_{r_{-} 1}=l_{r_{-} B}-l_{r_{-} A}$

$l_{r_{-} 2}=l_{r_{-} C}-l_{r_{-} B}$

$l_{r_{-} 3}=l_{r_{-} D}-l_{r_{-} C}$

For the cutting edge interpolation, it is assumed that the starting shape $W_{i}$ smoothly morphs into $W_{i+1}$ as tool wear progresses. In total, there are six wear profiles due to the six analysing steps. In a first step, a proportional distribution of points $Q_{i j}$ is applied on each of the three regionspecific arc lengths $l_{r_{-}}, l_{r_{-} 2}$ and $l_{r_{-} 3}$ of $W_{i}$. While the first index " $i$ " refers to that of the wear state, the second index " $j$ " describes the point number with respect to the direction of counting in positive $l_{r}$-direction, beginning in points $A, B$ and $C$ depending on the respective region. This distribution of points is exemplarily shown in Fig. $5 \mathrm{a}$ for R2 at $l_{\text {cut }}=5 \mathrm{~m}$ with a strongly enlarged point spacing due to visibility reasons. For the interpolation, a point spacing of $l_{s p}=0.5 \mu \mathrm{m}$ is used. In a next step, the same region-specific number of points used for $W_{i}$ is evenly distributed over the three regions 


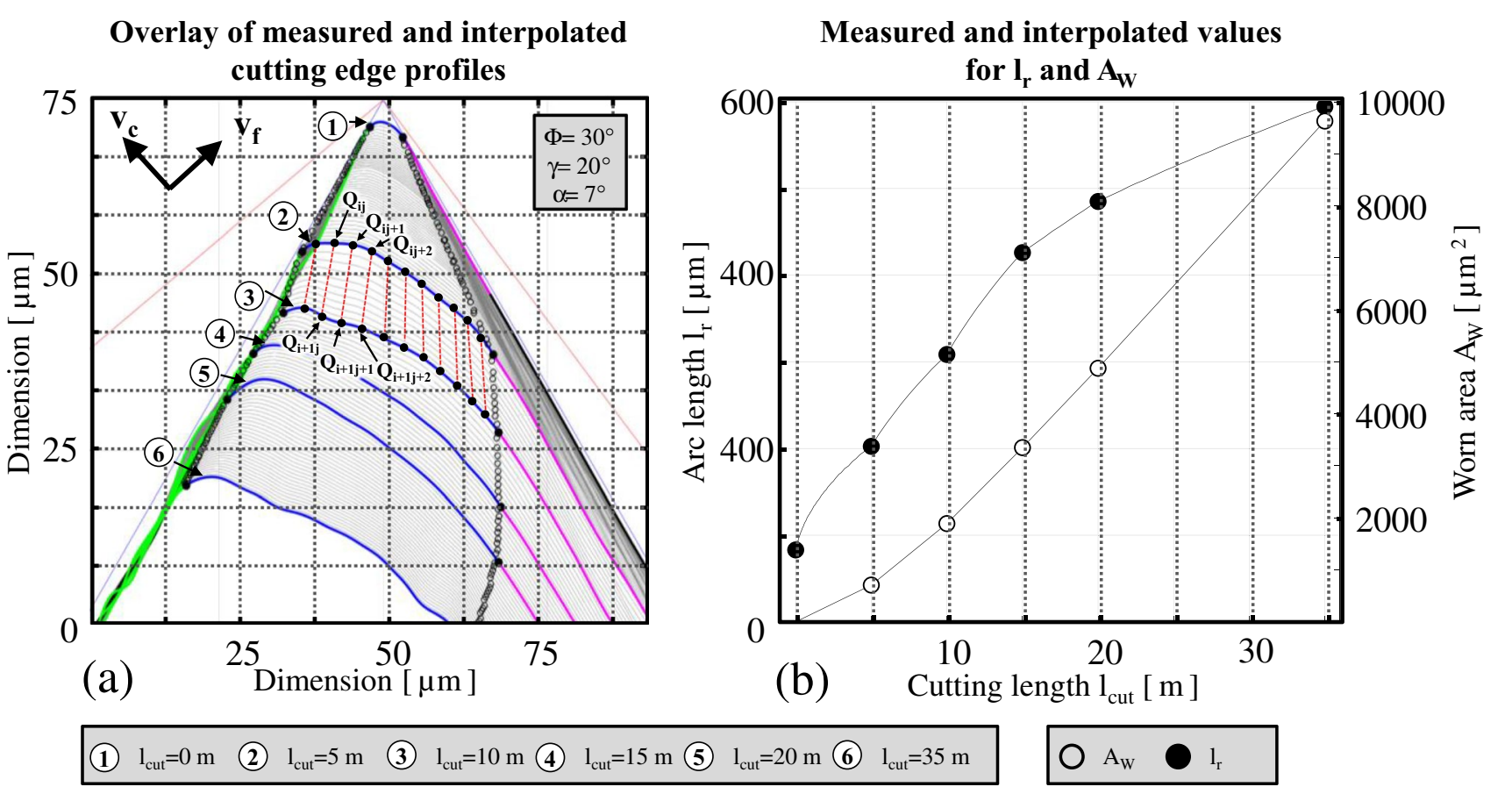

Fig. 5 (a) Exemplary overlay of measured and interpolated cutting edge profiles using a cutting length increment of $l_{\text {cut_int }}=0.5 \mathrm{~m}$, and (b) measured and interpolated values for the arc length $l_{r}$ and the worn area $A_{W}$ with respect to the cutting length

of the next known cutting edge profile $W_{i+1}$. For $l_{\text {cut }}=5 \mathrm{~m}$, this is the measured profile at $l_{\text {cut }}=10 \mathrm{~m}$. By connecting the corresponding points from each region on profile $W_{i}$ to the points on the subsequent profile $W_{i+1}$, individual pairs are formed as shown in Fig. 5a. It has to be mentioned that the orientations of these connecting lines are different to the directions of the wear vectors which are defined orthogonal to the starting shape.

As a first approximation, it is assumed that each point $Q_{i j}$ on $W_{i}$ follows the respective connecting line if tool wear progresses until it lies on $W_{i+1}$. Due to the proportional distributions of points for $W_{i}$ and $W_{i+l}$, this approach corresponds to a linear interpolation between both profiles, which is acceptable if the profile's change in geometry can be described by $2 \mathrm{D}$ translation and stretching. Although this approach provides a basis for the geometric interpolation between profiles, the interpolated profiles must be associated with a specific point in time or a specific cutting distance. This can be achieved by considering the measured worn area $A_{W}$ between $W_{i}$ and $W_{i+l}$. Assuming a linear trend, the worn area between consecutive profiles, both measured and interpolated, should follow the same trend in worn area that is seen between just the original measured profiles. Based on the experimental findings of Seeholzer et al. [20], it is assumed that the material removal rate between two measured profiles is constant. As exemplarily shown in Fig. 5a, new profiles between $W_{i}$ and $W_{i+1}$ are then determined in order of increasing the cutting distance by iteratively interpolating for new points between each of the point-pairs evaluated above. The interpolation ratio used for each new profile is iteratively adjusted such that the total worn area between $W_{i}$ and $W_{i+l}$ is equally divided between each profile. For example, if the worn area between two measured profiles is $50 \mu \mathrm{m}^{2}$ and four new profiles are desired, then the resulting worn area between each interpolated profile would be $10 \mu \mathrm{m}^{2}$. If the cutting distances associated to the two measured profiles are $l_{\text {cut }}=5 \mathrm{~m}$ and $l_{\text {cut }}=10 \mathrm{~m}$, then the cutting distances for the interpolated cutting edge profiles would be $l_{\text {cut }}=6 \mathrm{~m}$, $l_{\text {cut }}=7 \mathrm{~m}, l_{\text {cut }}=8 \mathrm{~m}$ and $l_{\text {cut }}=9 \mathrm{~m}$.

Figure 5a shows an overlay of an exemplary series of cutting edge profiles, where the measured and the interpolated cutting edge profiles are depicted. For this purpose, the worn tool material between consecutive measured cutting edge profiles is divided by their difference in cutting length, which yields the interval-specific material loss per metre cutting length. Subsequently, the number of interpolations between the respective measured profiles is chosen such that the material loss between two consecutive wear profiles, both measured and interpolated, equals to a cutting length interval of $l_{\text {cut_int }}=0.5 \mathrm{~m}$. The resulting refining of the wear data is sufficiently small to avoid the limitations of wear vectors mentioned before. Figure $5 \mathrm{~b}$ shows the measured and interpolated values of the arc length 
$l_{r}$ and the worn tool area $A_{W}$ with respect to the cutting length.

\subsubsection{Discussion of the calculated wear rate distributions}

In preparation for the analytical wear model, the WRD and its dependencies on the initial tool geometry, the fibre cutting angle and the cutting length have to be investigated. For this purpose, the wear vector approach introduced in Sect. 2.3 is applied on the interpolated and measured cutting edge profiles, where the interpolation strategy in Sect. 3.2.1 is used with a cutting length interval of $l_{\text {cut_int }}=0.5 \mathrm{~m}$. Figure 6 shows an overview of the resulting WRD for the tool $\mathrm{H}(10 / 14)$ after machining different fibre cutting angles after cutting lengths of $l_{\text {cut }}=0 \mathrm{~m}, l_{\text {cut }}=15 \mathrm{~m}$ and $l_{\text {cut }}=35 \mathrm{~m}$. In order to improve the comparability of the shown WRD with respect to the fibre cutting angle and the cutting length, the same maximum arc length on the $x$-axis is used for all plots. The scaling of the $y$-axis varies for the different fibre cutting angles but is constant for all plots of one specific value of $\Phi$. For each plotted WRD, the points $A, B, C, D$ and $W_{\max }$ are indicated and the colours green, blue and magenta are used for highlighting the regions R1, R2 and R3. Figure 7 shows the identical overview of the resulting WRD for the tools E (10/7), I (10/21) and L (20/14) in machining $\Phi=0^{\circ}$ in order to investigate the influence of the initial tool geometry on the characteristic of the WRD.

According to Figs. 6 and 7, some of the plotted WRD are characterised by a noticeable amount of fluctuation, particularly in R1 and R3 close to the contact points $A$ and $D$. This is due to the applied measuring strategy, where before interpolating between different cutting edge profiles

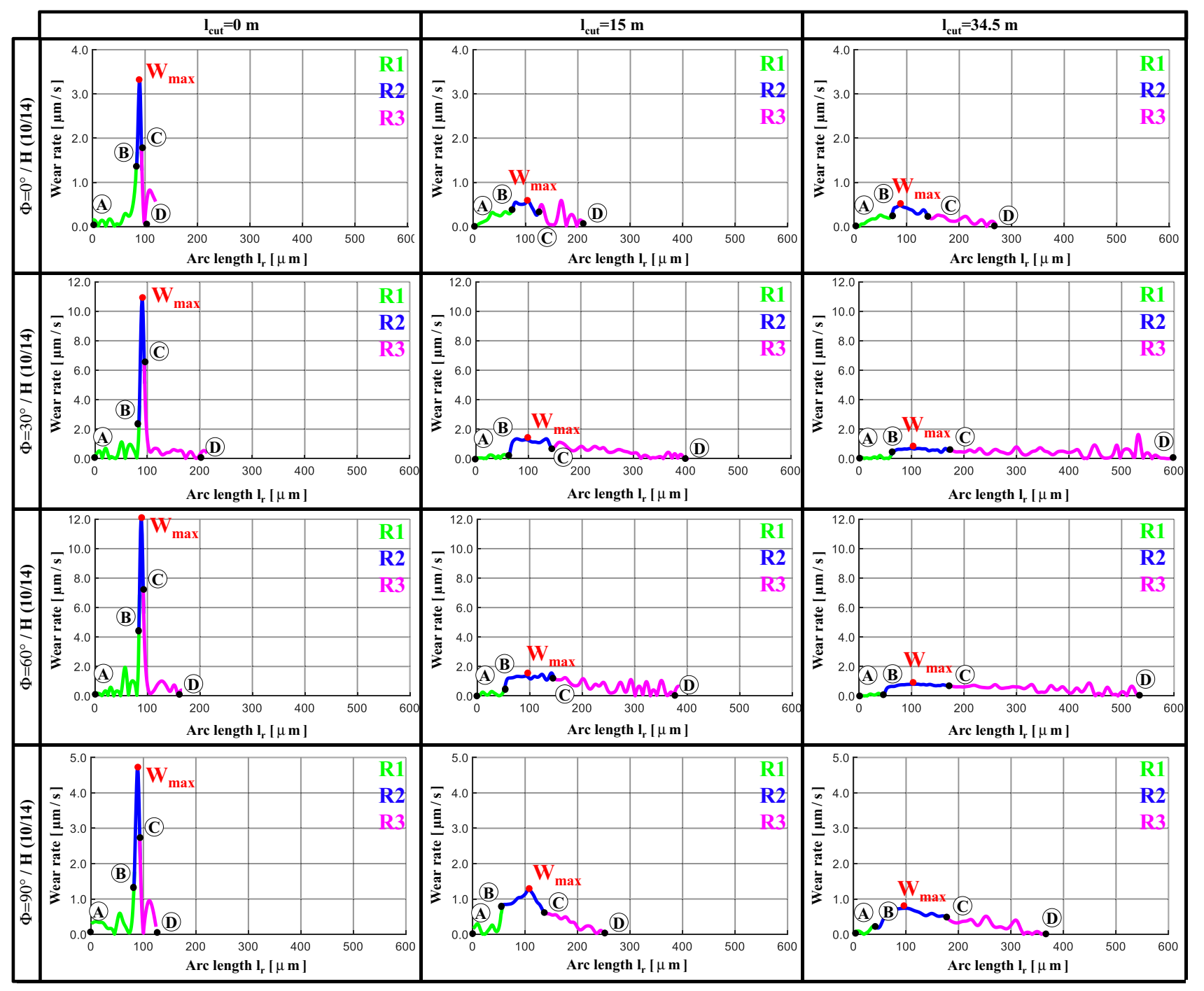

Fig. 6 Measured WRD for tool $\mathrm{H}(10 / 14)$ in machining $\Phi=0^{\circ}, \Phi=30^{\circ}, \Phi=60^{\circ}$ and $\Phi=90^{\circ}$ at cutting lengths of $l_{\text {cut }}=0 \mathrm{~m}, l_{\text {cut }}=15 \mathrm{~m}$ and $l_{\text {cut }}=34.5 \mathrm{~m}\left(v_{c}=90 \mathrm{~m} / \mathrm{min}, f=0.03 \mathrm{~mm} / \mathrm{rev}, l_{\text {cut_int }}=0.5 \mathrm{~m}\right)$ 


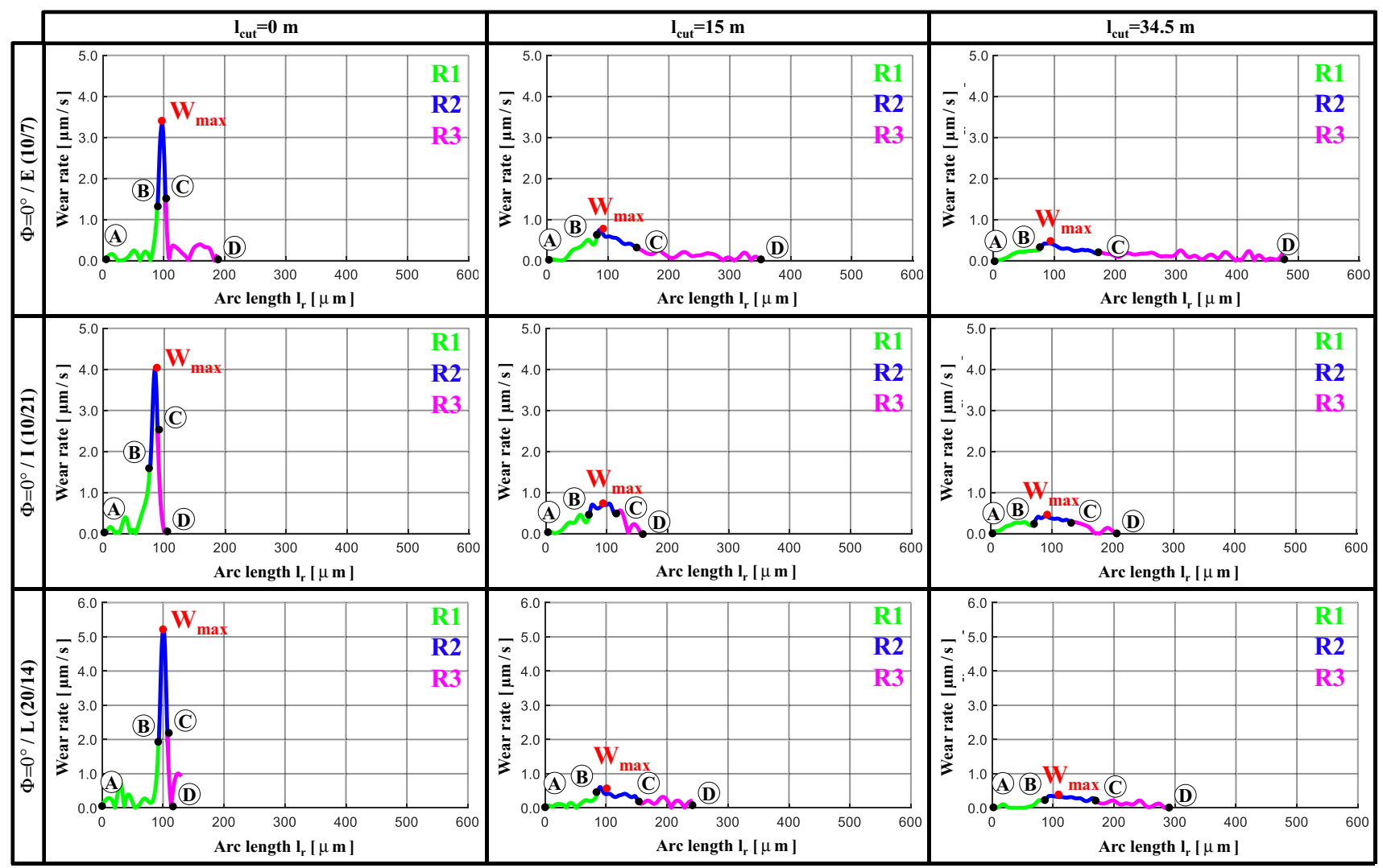

Fig. 7 Measured WRD for the tools E (10/7), I (10/21) and L (20/14) in machining $\Phi=0^{\circ}$ at cutting lengths of $l_{\text {cut }}=0 \mathrm{~m}, l_{\text {cut }}=15 \mathrm{~m}$ and $l_{\text {cut }}=34.5 \mathrm{~m}\left(v_{c}=90 \mathrm{~m} / \mathrm{min}, f=0.03 \mathrm{~mm} / \mathrm{rev}, l_{\text {cut_int }}=0.5 \mathrm{~m}\right)$

at different wear states, the measured profiles at $l_{\text {cut }}=0 \mathrm{~m}$, $l_{\text {cut }}=5 \mathrm{~m}, l_{\text {cut }}=10 \mathrm{~m}, l_{\text {cut }}=15 \mathrm{~m}, l_{\text {cut }}=20 \mathrm{~m}$ and $l_{\text {cut }}=35 \mathrm{~m}$ have to be aligned for the overlays. In this context, small misalignments between the measured profiles are transferred to the interpolated cutting edge profiles where the axial shifting between two consecutive profiles causes a distortion of the wear vectors. Therefore, the calculated magnitude of the wear vectors are characterised by a percentage error depending on the degree of the shifting error. Whereas this error in magnitude is negligible for most points along the active micro-geometry, it causes a noticeable amount of fluctuation in tool contact areas where the material removal rate is small as it is the case close to the points $A$ and $D$.

In the relatively new state of the cutting tool, occurring tool wear is particularly concentrated on R2 while the contributions of R1 and R3 to the total wear rate are negligible. As tool wear progresses, the peak value of the wear rate distribution $W_{\max }$ drops clearly, while simultaneously the WRD extends over a continuously increasing arc length. Nevertheless, the peak value $W_{\max }$ is always located in R2. According to Figs. 6 and 7, this characteristic flattening trend of the WRD is found for each tested combination of the initial tool geometry and the fibre cutting angle.
Furthermore, Figs. 6 and 7 reveal that the shape and size of the WRD and their changes with progressive tool wear are highly influenced by the initial tool geometry and the fibre cutting angle. Using the example of the tool geometry $\mathrm{H}(10 / 14)$, Fig. 6 shows that the extension of $l_{r}$ is the most intense for $\Phi=30^{\circ}$, followed by $\Phi=60^{\circ}, \Phi=90^{\circ}$ and $\Phi=0^{\circ}$. After a cutting length of $l_{\text {cut }}=35 \mathrm{~m}$, the total arc length in machining $\Phi=30^{\circ}$ is about twice as high as in machining $\Phi=0^{\circ}$. For these fibre cutting angles and the tool $\mathrm{H}(10 / 14)$, the difference in $W_{\max }$ is the largest in the relatively new state of the tool and decreases as tool wear progresses. Using the example of $\Phi=0^{\circ}$, the influence of the initial clearance angle on the WRD can be analysed by comparing the results of the tools E (10/7), H (10/14) and I (10/21) in Figs. 6 and 7. Accordingly, the extension of $l_{r}$ is more intense if a cutting insert with a smaller initial clearance angle is used. After a cutting length of $l_{c u t}=35 \mathrm{~m}$, the total arc length for the tool $\mathrm{E}(10 / 7)$ is nearly twice as high as for the tool $\mathrm{H}(10 / 14)$ and about 2.5 times higher than that measured for tool I (10/21). Analogous to the clearance angle, the influence of the initial rake angle on the WRD can be analysed by comparing the results of the tools $H(10 / 14)$ and $L$ (20/14). According to Figs. 6 and 7, in machining $\Phi=0^{\circ}$, the WRD of both tools are comparable for all three cutting lengths which indicates 
that the influence of the initial rake angle on the WRD and its wear-related change is negligible.

Although the WRD show clear differences in terms of shape and size with respect to different combinations of the initial tool geometry and the fibre cutting angle, the following general commonality can be formulated: Beginning in point $A$ at $l_{r_{-} A}=0$, the wear rate increases evenly along the tool shape with increasing $l_{r}$, reaches its maximum between the points $B$ and $C$ in R2 and subsequently decreases evenly to zero in direction to point $D$.

Another commonality is the fact that the relative position of $W_{\max }$ with respect to the normalised arc length of $\mathrm{R} 2$ is comparable for all tested tool geometries and their wear states, but changes clearly, if the fibre cutting angle is varied. This coincides with the experimental results of Seeholzer et al. [20], where the wear-related change of R2 in terms of $l_{\gamma}$ and $l_{\alpha}$ mainly depends on the fibre cutting angle while the influence of the initial tool geometry is negligible. As a result, a representative location for the maximum of the WRD can be determined for each tested fibre cutting angle $\Phi=0^{\circ}, \Phi=30^{\circ}$, $\Phi=60^{\circ}$ and $\Phi=90^{\circ}$ as shown in Fig. 8. This representative maximum is represented by $W_{\text {max }{ }_{-} \text {rep }}$ and its relative location can be described by the parameters $l_{r_{-} \_ \text {left }}$ to $l_{r_{-} 2}$ representing the arc length between the points $B$ and $W_{\text {max_rep }}$ and the arc length of R2. In this context, the parameter $n_{w}$ is introduced and is defined as the ratio of $l_{r_{-} 2 \text { left }}$ to $l_{r_{-} 2}$.

Based on the experimental results provided by Seeholzer et al. [20], a representative value of $n_{w}$ can be determined for each tested fibre cutting angle: $n_{w 0^{\circ}}, n_{w 30^{\circ}}, n_{w 60^{\circ}}, n_{w 90^{\circ}}$. These experimentally determined values are summarised in Table 3 . Based on the values in Table 3, the arc length of $W_{\text {max _rep }}$ represented by $l_{r_{-} W \max }$ can be calculated.

$l_{r_{-} W \max }=l_{r_{-} 1}+n_{w_{-} i} * l_{r_{-} 2} \quad\left(i=0^{\circ}, 30^{\circ}, 60^{\circ}, 90^{\circ}\right)$
Table 3 Experimentally determined values for $n_{w 0^{\circ}}, n_{w 30^{\circ}}, n_{w 60^{\circ}}$ and $n_{w 90^{\circ}}$ based on the orthogonal machining experiments [20]

\begin{tabular}{lll}
\hline Parameter $(-)$ & Fibre cutting angle $\Phi\left(^{\circ}\right)$ & Value $(-)$ \\
\hline$n_{w 0^{\circ}}$ & 0 & 0.2 \\
$n_{w 30^{\circ}}$ & 30 & 0.3 \\
$n_{w 60^{\circ}}$ & 60 & 0.5 \\
$n_{w 90^{\circ}}$ & 90 & 0.3 \\
\hline
\end{tabular}

\section{Wear modelling approach}

The wear modelling approach presented in this section is based on the fundamental assumption that the WRD associated to an arbitrary wear state $W_{i}$ of the cutting edge can be approximated as a function of the corresponding five wear parameters $\gamma^{*}, \alpha^{*}, l_{\gamma}, l_{\alpha}$ and $b_{c}$. Conversely, this means that if the five wear parameters are known for a specific wear state $W_{i}$, the corresponding WRD along the arc length can be reproduced mathematically. This calculated WRD can then be used in order to update the wear state form $W_{i}$ to $W_{i+1}$ using the concept of wear vectors introduced in Sect. 2 in a reverse order. Subsequently, the five wear parameters can be calculated for the updated wear state $W_{i+1}$ which then forms the basis for a new calculation of the WRD. Providing the step size between two consecutive wear states $W_{i}$ and $W_{i+1}$ to be sufficiently small, this procedure enables to simulate the wear-related change of the active micro-geometry during machining.

\subsection{Parameterisation of the wear rate distribution along the cutting edge}

The simplest approach for a shape parameterisation is to define one higher-order function that allows the approximation of the

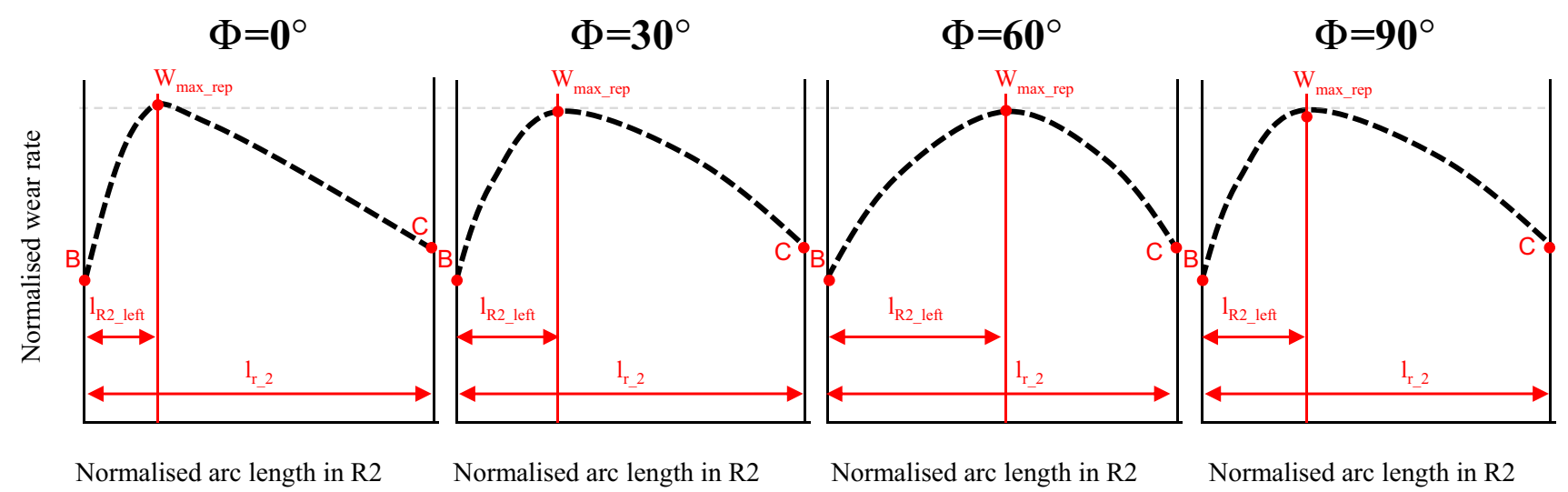

Fig. 8 Schematic illustration of the representative maximum $W_{\text {max_resp }}$ for $\Phi=0^{\circ}, \Phi=30^{\circ}, \Phi=60^{\circ}, \Phi=90^{\circ}$ and its localisation using the parameters $l_{r_{-} \_ \text {left }}$ to $l_{r_{-} 2}$ 
Approximation WRD with general sub-functions $\mathbf{r}_{1}, \mathbf{r}_{2}, \mathbf{r}_{3}$

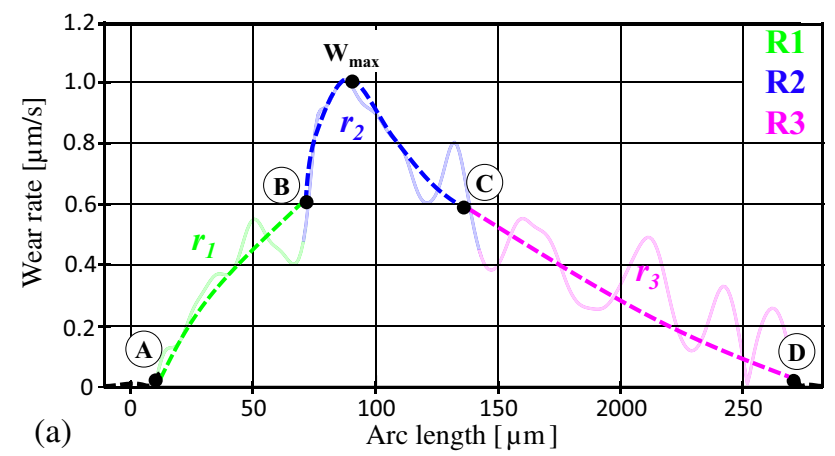

Approximation WRD with the "line-curve-line" approach

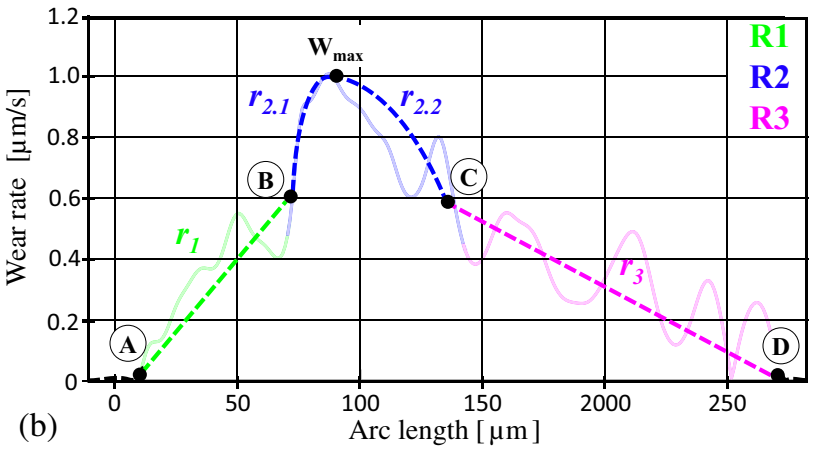

Fig. 9 Approximation of an arbitrary WRD using (a) three general polynomial functions $r_{1}, r_{2}$ and $r_{3}$ and (b) using the "line - curve line" approach with $r_{1}, r_{2.1}, r_{2.2}$ and $r_{3}$

entire WRD at once. However, this approach is not constructive since it means that the WRD with its variations depending on the initial tool geometry, the fibre cutting angle and the cutting length have to be described by one single function, which therefore would be rather complex. As an alternative, the general shape of a WRD can be expressed as a piecewise function with three sub-functions $r_{1}, r_{2}$ and $r_{3}$, one for each micro-geometry region. This is schematically visualised in Fig. 9a. Initially, the three region-specific wear rate functions $r_{1}, r_{2}$ and $r_{3}$ can be expressed as general polynomial functions, where the parameters $n, p$ and $q$ represent the order of the polynomials.

$$
\begin{aligned}
& r_{1}\left(l_{r}\right)=\sum_{k=0}^{n} a_{k} l_{r}^{k} \quad n \geq 0 ; l_{r_{-} A} \leq l_{r} \leq l_{r_{-} B} \\
& r_{2}\left(l_{r}\right)=\sum_{m=0}^{p} b_{m} l_{r}^{m} \quad p \geq 0 ; l_{r_{-} B} \leq l_{r} \leq l_{r_{-} C} \\
& r_{3}\left(l_{r}\right)=\sum_{u=0}^{q} c_{u} l_{r}^{u} \quad q \geq 0 ; l_{r_{-} C} \leq l_{r} \leq l_{r_{-} D}
\end{aligned}
$$

On the one hand, high-order polynomials are desirable since they increase the degree of representable geometry features. On the other hand, this means that more boundary conditions have to be provided in order to determine the unknown coefficients $a_{k}, b_{m}$ and $c_{u}$. In order to find appropriate polynomial functions for the three regions while simultaneously keep the number of required boundary conditions as low as possible, the basic functions for $r_{1}, r_{2}$ and $r_{3}$ have to be adjusted to the characteristic shape of the WRD in R1, $\mathrm{R} 2$ and R3. For this purpose and based on the experimental results presented in Sect. 3.2.2, the following assumptions are made: The shape of the WRD between the points $A$ and $B$ in R1 can be approximated with a line using a 1st-order polynomial. The shape of the WRD between the points $B$ and $W_{\max }$ in $\mathrm{R} 2$ can be approximated with a curve using a 2nd-order polynomial. The shape of the WRD between the points $W_{\max }$ and $C$ in $\mathrm{R} 2$ can be approximated with a curve using a 2nd-order polynomial. The shape of the WRD between the points $C$ and $D$ in R 3 can be approximated with a line using a 1st-order polynomial. The resulting shape approximation in terms of a "line - curve - line" approach is visualised in Fig. 9b.

With the "line - curve - line" approach, the wear rate functions given in Eq. (5) can then be simplified as follows:

$r_{1}\left(l_{r}\right)=a_{1} l_{r}+a_{0}$

$r_{2.1}\left(l_{r}\right)=b_{2} l_{r}^{2}+b_{1} l_{r}+b_{0}$

$r_{2.2}\left(l_{r}\right)=c_{2} l_{r}^{2}+c_{1} l_{r}+c_{0}$

$r_{3}\left(l_{r}\right)=d_{1} l_{r}+d_{0}$

$$
\begin{gathered}
l_{r_{-} A} \leq l_{r} \leq l_{r_{-} B} \\
l_{r_{-} B} \leq l_{r} \leq l_{r_{-} W \max } \\
l_{r_{-} \text {Wmax }} \leq l_{r} \leq l_{r_{C}} \\
l_{r_{-} C} \leq l_{r} \leq l_{r_{-} D}
\end{gathered}
$$

According to Fig. 9b, the sub-functions $r_{1}, r_{2.1}, r_{2.2}$ and $r_{3}$ have to be coherent with respect to the total WRD, which means that the $C^{0}$ continuity has to be fulfilled at the transition points $B, W_{\max }$ and $C$.

$$
\begin{aligned}
& r_{1}\left(l_{r_{-} B}\right)=r_{2.1}\left(l_{r_{-} B}\right) \\
& r_{2.1}\left(l_{r_{-} \text {Wmax }}\right)=r_{2.2}\left(l_{r_{-} \text {Wmax }}\right) \\
& r_{2.2}\left(l_{r_{-} C}\right)=r_{3}\left(l_{r_{-} C}\right)
\end{aligned}
$$

As shown in Fig. 9b, the point $W_{\max }$ represents the vertex for the parabolas of $r_{2.1}$ and $r_{2.2}$. Consequently, the polynomial functions $r_{2.1}$ and $r_{2.2}$ in Eq. (6) can be simplified by using the vertex form of a parable, where one coefficient and the coordinates of the vertex, represented by $X_{V}$ and $Y_{V}$, have to be known. In accordance with Fig. 3, the vertex coordinates $X_{V}$ and $Y_{V}$ are identical to $l_{r_{-} \text {Wmax }}$ and $z_{r_{-} \text {Wmax }}$, which means that the polynomial functions $r_{2.1}$ and $r_{2.2}$ can be written as follows:

$\begin{array}{ll}r_{2.1}\left(l_{r}\right)=b_{3}\left(l_{r}-l_{r_{-} W \max }\right)^{2}+z_{r_{-} W \max } & l_{r_{-} B} \leq l_{r} \leq l_{r_{-} W \max } \\ r_{2.2}\left(l_{r}\right)=c_{3}\left(l_{r}-l_{r_{-} W \max }\right)^{2}+z_{r_{-} W \max } & l_{r_{-} W \max } \leq l_{r} \leq l_{r_{-} C}\end{array}$ 
Subsequently, the unknown coefficients $b_{3}$ and $c_{3}$ can be calculated using the information that in accordance with Eq. (7), the points $B$ and $C$ have to be points of $r_{2.1}$ and $r_{2.2}$, respectively.

$b_{3}=\frac{\left(z_{r_{-} B}-z_{r_{-} W \max }\right)}{\left(l_{r_{-} B}-l_{r_{-} W \max }\right)^{2}}$
$c_{3}=\frac{\left(z_{r_{-} C}-z_{r_{-} W \max }\right)}{\left(l_{r_{-} C}-l_{r_{-} W \max }\right)^{2}}$

By definition, the WRD is bounded by the contact points $A$ and $D$, where the wear vector's magnitude is zero. Therefore, the parameters $\mathrm{z}_{r_{-} A}$ and $\mathrm{z}_{r_{-} D}$, representing the functional values of the WRD for the contact points $A$ and $D$, have to be zero as well. Considering that $l_{r_{-} A}$ is identical to zero by definition and $B$ is a point of $r_{1}$, the coefficients $a_{0}$ and $a_{1}$ can be determined as follows:

$a_{0}=0$

$a_{1}=\frac{z_{r_{-} B}}{l_{r_{-} B}}$

Analogous, the coefficients $d_{0}$ and $\mathrm{d}_{1}$ for $\mathrm{r}_{3}$ can be determined under consideration that $C$ is a point of $\mathrm{r}_{3}$.

$d_{0}=-\frac{z_{r_{-} C} l_{r_{-} D}}{\left(l_{r_{r}}-l_{r_{-}}\right)}$
$d_{1}=\frac{\left(z_{r_{-} C} C\right.}{\left(l_{r_{-} C}-l_{r_{-} D}\right)}$

By combining Eqs. (7), (8), (9), (10) and (11), the wear rate functions in Eq. (6) can be written as follows: mechanisms. Both parameters are used as fitting constants that have to be determined individually for each machining situation.

$\frac{d W}{d t}=C_{1} \sigma_{n} v_{s} e^{\left(\frac{C_{2}}{T_{\text {cut }}}\right)}$

According to Eq. (13), the wear rate for each point in the contact region and hence the corresponding WRD can be calculated if the values of the cutting temperature, the normal stress and the sliding velocity as well as the material- and process-specific fitting constants are known as functions of $l_{r}$. Especially in machining CFRP with its anisotropic material properties and brittle fracture behaviour, the complete analytical determinations of $T_{c u t}, \sigma_{n}$ and $v_{s}$ as functions of $l_{r}$ along the contact region are challenging. For this reason, two fundamental assumptions are made in order to reduce the complexity:

- The influence of the process temperature on the tool wear rate is neglected: According to Wang et al. [19], tool wear in machining CFRP is predominated by mechanical abrasion while thermal wear effects play minor roles due to the comparatively low process temperatures. As a first approach, the influence of the process temperature on the wear rate is neglected.

- Introduction of the mean wear rates: First, the worn area between two consecutive cutting edge profiles $A_{W}$ is divided among the three regions $\mathrm{R} 1, \mathrm{R} 2$ and $\mathrm{R} 3$

$r_{1}\left(l_{r}\right)=\frac{z_{r-B}}{l_{r-B}} l_{r}$

$r_{2.1}\left(l_{r}\right)=\frac{\left(z_{r_{-}-B}-z_{r_{-}-\max }\right)}{\left(l_{r_{-} B}-l_{r_{-} W \max }\right)^{2}}\left(l_{r_{-}}-l_{r_{-} W \max }\right)^{2}+z_{r_{-} W \max }$

$l_{r_{-} A} \leq l_{r} \leq l_{r_{-} B}$

$r_{2.2}\left(l_{r}\right)=\frac{\left(z_{r_{-}-}-z_{r_{-} W \max }\right)}{\left(l_{r_{-} C}-l_{r_{-} W \max }\right)^{2}}\left(l_{r}-l_{r_{-} W \max }\right)^{2}+z_{r_{-} W \max }$

$l_{r_{-} B} \leq l_{r} \leq l_{r_{-} \text {Wmax }}$

$l_{r_{-} W \max } \leq l_{r} \leq l_{r_{-} C}$

$r_{3}\left(l_{r}\right)=z_{r_{-} C} \frac{\left(l_{r}-l_{r_{-} D}\right)}{\left(l_{r_{-} C}-l_{r_{-} D}\right)}$

$l_{r_{-} C} \leq l_{r} \leq l_{r_{-} D}$

\subsection{Specification of the wear rate}

Assumed that the contact points $A, B, C$ and $D$ and their positions along the arc length $l_{r_{-} A}, l_{r_{-} B}, l_{r_{-} C}$ and $l_{r_{-} C}$ are known for a specific wear state, the value of $l_{r_{-} W \max }$ can be calculated according to Eq. (4). This means that only the values of $z_{r_{-} B}, z_{r_{-} W \max }$ and $z_{r_{-} C}$ are missing before the corresponding WRD can be approximated based on the "line - curve - line" approach introduced in Sect. 4.1. In order to determine these values as functions of the active microgeometry, an approach similar to that of Usui et al. [37] is applied. Usui et al. [37] established a mathematical relation between the cutting temperature $T_{c u}$, the normal stress $\sigma_{n}$, the sliding velocity $v_{s}$ and the resulting wear rate. The parameters $C_{1}$ and $C_{2}$ describe the surface activity and the activation energy that is required to start the wear which yields the region-specific material losses represented by $A_{W_{-} l}, A_{W_{\_} 2}$ and $A_{W_{\_} 3}$. Subsequently, for each region, a mean wear rate is formulated based on Eq. (2), which describes the region-specific mean material loss per region-specific arc length per unit of time.

$\bar{w}_{1}=\frac{A_{W_{-} 1}}{l_{r_{-} 1} t}$
$\bar{w}_{2}=\frac{A_{W_{-}}}{l_{r_{-} 2} t}$
$\bar{w}_{3}=\frac{A_{W_{-}}}{l_{r_{-} 3} t}$

In a next step, it is assumed that the region-specific mean wear rates can be approximated as functions of the corresponding values of the region-specific mean contact stresses $\bar{\sigma}_{k_{-} 1}, \bar{\sigma}_{k_{-} 2}$ and $\bar{\sigma}_{k_{-} 3}$ and the mean sliding velocities $\bar{v}_{s_{-} 1}, \bar{v}_{s_{-} 2}$ and $\bar{v}_{s_{-} 3}$. 
$\bar{w}_{1}=A_{1} \bar{\sigma}_{n \_1} \bar{v}_{S_{-} 1}$

$\bar{w}_{2}=A_{2} \bar{\sigma}_{n \_2} \bar{v}_{s_{-} 2}$

$\bar{w}_{3}=A_{3} \bar{\sigma}_{n \_3} \bar{v}_{s_{-} 3}$

Whereas the parameters $A_{1}, A_{2}$ and $A_{3}$ are fitting parameters that have to be determined experimentally, the regionspecific mean contact stresses and sliding velocities can be derived mathematically as described in the following Sects. 4.2.1 and 4.2.2.

\subsubsection{Approximation of a region-specific mean contact stresses}

As a first approach, the mean contact stress in each region can be approximated by dividing the region-specific contact force $\bar{F}_{k_{-} i}(i=1,2,3)$ by the corresponding arc length $l_{r_{-} i}$ $(i=1,2,3)$.

$\bar{\sigma}_{k \_i}=\frac{\bar{F}_{k_{-} i}}{l_{r_{-} k}}$ with $i=1,2,3$

For the calculation of the region-specific mean contact force $\bar{F}_{n \_k}$ in Eq. (16), the analytical force modelling approach presented by Voss et al. [8] is used. This analytical force model uses the five wear parameters $\gamma^{*}, \alpha^{*}, l_{\gamma}, l_{\alpha}$ and $b_{c}$ for an arbitrary active micro-geometry as input data and yields the corresponding cutting and thrust force components $F_{c_{-} i}(i=1,2,3)$ and $F_{t_{-} i}(i=1,2,3)$ for each of the three regions. For the sake of completeness, a list of the main formulas for the determination of region-specific force components depending on the three fibre cutting intervals $I 1$ : $\Phi=0^{\circ}=180^{\circ}, I 2: 15^{\circ} \leq \Phi \leq 75^{\circ}$ and $I 3: \Phi=90^{\circ}$ is provided in the Appendix of this work. A list of force model-specific parameters and their descriptions in presented in Table 7 in the Appendix. Once the region-specific cutting and thrust force components are known for an arbitrary wear state, the corresponding mean normal forces $\bar{F}_{k_{-} i}(i=1,2,3)$ can be approximated as their resultant forces.

$\bar{F}_{k_{-} i}=\sqrt{F_{c_{-} i}{ }^{2}+F_{t_{-} i}^{2}}$ with $i=1,2,3$

\subsubsection{Approximation of a region-specific mean sliding velocity}

Analogous to the mean contact stresses, mean sliding velocities for R1, R2 and R3 have to be formulated. The sliding velocity describes the relative motion within the contact zone of the cutting tool and the CFRP material and depends on the effective cutting velocity and the tool shape.

For machining situations, where the cutting velocity is much higher than the feed velocity $\left(v_{c} \gg v_{f}\right)$, the effective cutting direction is nearly parallel to the cutting velocity direction. Therefore, as a first approximation, the influence of the feed velocity on the mean sliding velocity is neglected. The sliding velocity in $\mathrm{R} 1$ is driven by the evacuation of produced chips along the tool's rake face. As a first approach, the resulting relative velocity between the CFRP material and the tool surface on the rake face can be approximated with the cutting velocity as stated by $\mathrm{Li}$ et al. [38]. In order to approximate the mean sliding velocity in R2, it is assumed that the machined CFRP material is pressed along the arc length of $\mathrm{R} 2$ while the cutting tool covers a distance of $l_{\alpha}$ in cutting velocity direction. Since the duration theoretically has to be identical for both travel paths, the mean sliding velocity in $\mathrm{R} 2$ can be determined as function of $l_{\alpha}, l_{r_{-} 2}$ and $v_{c}$. For the modelling approach, the ratio of $l_{r_{-} 2} / l_{\alpha}$ is squared as it was found experimentally to fit better with the mean wear trends than leaving the ratio unsquared. After passing the contact point $C$, the compressed CFRP material rubs along the flank face, where analogous to $\mathrm{R} 2$, the mean sliding velocity can be approximated as function of $l_{r_{-} 3}, v_{c}$ and the distance between the points $C$ and $D$ in cutting velocity direction which is represented by $l_{3}$.

$$
\begin{aligned}
& \bar{v}_{s_{-} 1}=v_{c} \\
& \bar{v}_{s_{-} 2}=v_{c}\left(\frac{l_{r_{-}}}{l_{\alpha}}\right)^{2} \\
& \bar{v}_{s_{-} 3}=v_{c}\left(\frac{l_{r_{-} 3}}{l_{3}}\right) \text { with } l_{3}=l_{r_{-} 3} \cos \left(\alpha^{*}\right)
\end{aligned}
$$

By considering the region-specific formulations for the mean contact stresses in Eq. (16) and sliding velocities in Eq. (18), the corresponding mean wear rates in Eq. (15) can be rewritten as follows:

$\bar{w}_{1}=\frac{A_{1} \bar{F}_{k_{-1}} v_{c}}{l_{l_{1} 1}}$

$\bar{w}_{2}=\frac{A_{2} \bar{F}_{k_{-}} v_{c}}{{\underline{r_{-} 2}}_{r_{-}}}\left(\frac{l_{r_{-} 2}}{l_{\alpha}}\right)^{2}$

$\bar{w}=\frac{A_{3} \bar{F}_{k_{-}} v_{c}}{l_{r_{-} 3}}\left(\frac{1}{\cos \left(\alpha^{*}\right)}\right)$

In a next step, the approximated mean wear rates $\bar{w}_{1}, \bar{w}_{2}$ and $\bar{w}_{3}$ are used to calculate the values of $z_{r_{-} B}, z_{r_{-} \text {Wmax }}$ and $z_{r_{-} C}$. Since the wear rate at point $A$ is zero and its distribution between the contact points $A$ and $B$ is assumed linear according to Fig. 9b, the wear rate at the contact point $B$ is equal to twice the mean wear rate in $\mathrm{R} 1$.

$z_{r_{-} B}=2 \bar{w}_{1}=2 A_{1} \frac{\bar{F}_{k_{-} 1}}{l_{r_{-} 1}} v_{c}$

Based on Eq. (20), the formulation of $r_{l}$ in Eq. (12) can be rewritten as follows:

$r_{1}\left(l_{r}\right)=2 A_{1} \frac{\bar{F}_{k_{-} 1}}{l_{r_{-} 1}} v_{c} \frac{1}{l_{r_{-} B}} l_{r} \quad l_{r_{-} A} \leq l_{r} \leq l_{r_{-} B}$ 
Table 4 Measured values for the bouncing back height for tool $\mathrm{H}(10 / 14)$ in machining $\Phi=0^{\circ}, \Phi=30^{\circ}, \Phi=60^{\circ}$ and $\Phi=90^{\circ}$ and for the tools $\mathrm{E}$ (10/7), I (10/21) and L (20/14) in machining $\Phi=0^{\circ}$ based on [20]

\begin{tabular}{|c|c|c|c|c|c|c|}
\hline $\begin{array}{l}\text { Tool } \\
(-)\end{array}$ & $\begin{array}{l}\text { Fibre cutting } \\
\text { angle } \Phi \\
\left({ }^{\circ}\right)\end{array}$ & $\begin{array}{l}b_{c} \text { at } 1_{c u t}=5 \mathrm{~m} \\
(\mu \mathrm{m})\end{array}$ & $\begin{array}{l}b_{c} \text { at } l_{c u t}=10 \mathrm{~m} \\
(\mu \mathrm{m})\end{array}$ & $\begin{array}{l}b_{c} \text { at } l_{c u t}=15 \mathrm{~m} \\
(\mu \mathrm{m})\end{array}$ & $\begin{array}{l}b_{c} \text { at } l_{c u t}=20 \mathrm{~m} \\
(\mu \mathrm{m})\end{array}$ & $\begin{array}{l}b_{c} \text { at } l_{c u t}=35 \mathrm{~m} \\
(\mu \mathrm{m})\end{array}$ \\
\hline H (10/14) & 0 & 4 & 6.7 & 7.5 & 10 & 11.6 \\
\hline $\mathrm{H}(10 / 14)$ & 30 & 17.6 & 20 & 22.4 & 24.4 & 33.4 \\
\hline $\mathrm{H}(10 / 14)$ & 60 & 14 & 16.3 & 19.2 & 21 & 22 \\
\hline H (10/14) & 90 & 7 & 8 & 8.3 & 12 & 14 \\
\hline $\mathrm{E}(10 / 7)$ & 0 & 6 & 8.4 & 9.3 & 13.5 & 15.9 \\
\hline I (10/21) & 0 & 3 & 4.7 & 5 & 7 & 9.2 \\
\hline L (20/14) & 0 & 4.1 & 6.2 & 7 & 9 & 11.1 \\
\hline
\end{tabular}

Analogous to the procedure in $\mathrm{R} 1$, the wear rate at the contact point $C$ is equal to twice the mean wear rate in $\mathrm{R} 3$.

$$
z_{r_{-} C}=2 \bar{w}_{3}=2 A_{3} \frac{\bar{F}_{k_{-} 3}}{l_{r_{-} 3}} v_{c}\left(\frac{1}{\cos \left(\alpha^{*}\right)}\right)
$$

Fig. 10 Schematic illustration of the implementation strategy of the analytical wear rate model
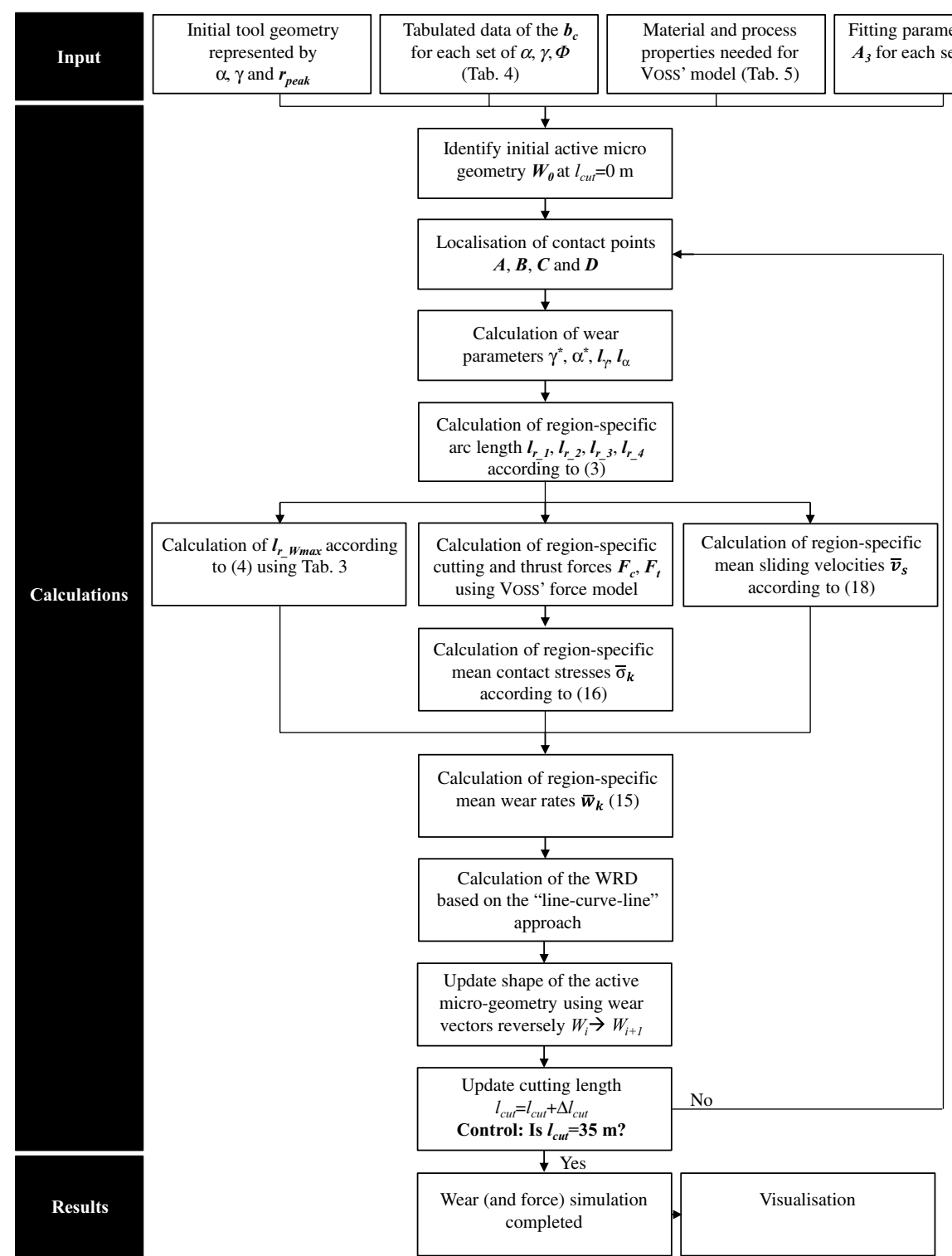
Table 5 List of material and process parameters and fitting constants used for Voss's analytical force model [8] based on $[15,17,18,39-41]$

\begin{tabular}{llll}
\hline Carbon fibre - IMA-12 k & \multicolumn{3}{l}{ Process/Model } \\
\hline Young's Modulus (axial) $-E_{f 1}$ & $297 \mathrm{GPa}$ & Friction coefficient $-\mu$ & 0.12 \\
Young's Modulus (transv.) $-E_{f 2}$ & $15 \mathrm{GPa}$ & Parameter matrix slipping $-\xi$ & 0.02 \\
Shear modulus $-G_{f}$ & $8.96 \mathrm{GPa}$ & Parameter interfacial bonding $-\eta$ & 1.98 \\
Poisson's ratio $-v_{f}$ & 0.2 & Winkler constant $-k_{b}$ & $115 \mathrm{GPa}$ \\
Fibre radius $-r_{f}$ & $3.5 \mu \mathrm{m}$ & & \\
Tensile strength $-\sigma_{T_{f} f 0}$ & $4.9 \mathrm{GPa}$ & & \\
Volume fraction $-V_{f}$ & $59.2 \%$ & & \\
Epoxy matrix $-\mathbf{M 2 1 / 3 4 \%}$ & & Fitting variables & \\
Young's Modulus $-E_{m \_} 0$ & $3.5 \mathrm{GPa}$ & Correction factor micro-buckling $-K_{f}$ & 0.33 \\
Shear modulus $-G_{m}$ & $1.02 \mathrm{GPa}$ & Height sub-region 2.2 $-H_{2.2}$ & $2.625 \mu m$ \\
Poisson's ratio $-v_{m}$ & 0.318 & Young's Modulus CFRP region $3-E_{c_{-} 3}$ & $17 \mathrm{GPa}$ \\
Shear strength $-S_{m}$ & $62 \mathrm{MPa}$ & Factor tensile fibre strength $-K_{\sigma_{-} T f}$ & 3.5 \\
& & Factor Young's Modulus matrix $-K_{E m}$ & 3 \\
& & Critical deflection $-\omega_{Z \prime \prime} c r$ & $0.26 \mu m$ \\
\hline
\end{tabular}

Considering Eq. (22), the formulation of $r_{2}$ in Eq. (12) can be rewritten as follows:

$r_{3}\left(l_{r}\right)=2 A_{3} \frac{\bar{F}_{k \_3}}{l_{r_{-} 3}} v_{c}\left(\frac{1}{\cos \left(\alpha^{*}\right)}\right) \frac{\left(l_{r}-l_{r_{-} D}\right)}{\left(l_{r_{-} C}-l_{r_{-} D}\right)} \quad l_{r_{-} C} \leq l_{r} \leq l_{r_{-} D}$

In order to calculate the value of $z_{r_{-} W \max }$, it can be used that the calculated mean wear rate in $\mathrm{R} 2$ has to be identical to that obtained with the two parables in the WRD while the horizontal position of $W_{\max }$ is predefined by the value of $n_{w}$.

$z_{r_{-} \text {Wmax }}=\frac{\bar{w}_{2}-\left(\frac{z_{r_{B} B} n_{w}}{3}\right)-\left(\frac{z_{r_{-} C}\left(1-n_{w}\right)}{3}\right)}{1-\frac{n_{w}}{3}-\frac{1-n_{w}}{3}}$

\section{Simulation design and procedure}

According to Sect. 4, the WRD of an arbitrary active microgeometry can be calculated if the corresponding five wear parameters $\gamma^{*}, \alpha^{*}, l_{\gamma}, l_{\alpha}$ and $b_{c}$ and the fitting parameters $A_{l}$, $A_{2}$ and $A_{3}$ are known. In the new state of a cutting tool, the initial values of the rake angle $\gamma$ and the clearance angle $\alpha$ are known due to the tool specifications. Furthermore, the major and minor semi-axes $l_{\alpha}$ and $l_{\gamma}$ are equal to the peak radius $r_{\text {peak }}$ of the tool which is known as well. In contrast, the fifth wear parameter, represented by the bouncing back height $b_{c}$, is not known since it is not a setting parameter but the result of complex elastoplastic deformations in the contact region. In order to consider the bouncing back height in the wear model, the experimental results in [20] are used in terms of the values of $b_{c}$ at the five different cutting lengths: $l_{\text {cut }}=5 \mathrm{~m}, l_{\text {cut }}=10 \mathrm{~m}, l_{\text {cut }}=15 \mathrm{~m}, l_{\text {cut }}=20 \mathrm{~m}, l_{\text {cut }}=35 \mathrm{~m}$. For the sake of completeness, Table 4 shows a list of the evaluated bouncing back heights for the tool $\mathrm{H}(10 / 14)$ in machining $\Phi=0^{\circ}, \Phi=30^{\circ}, \Phi=60^{\circ}$ and $\Phi=90^{\circ}$ and for the tools $\mathrm{E}$ (10/7), I (10/21) and L (20/14) in machining $\Phi=0^{\circ}$. A linear interpolation is applied in order to interpolate the values of $b_{c}$ between the measured cutting lengths resulting in an approximation of the bouncing back height as a tabulated data base with respect to the cutting length.

For the integration of the wear rate model, a forward Euler method is used. According to the schematic illustration in Fig. 10, the simulation is implemented as an iterative process, where based on the initial state of the cutting edge profile $W_{O}$ at $l_{c u t}=0 \mathrm{~m}$, all future worn states of a cutting edge profile $W_{i}(i>0)$ are calculated loop wise as explained in the following.

According to Fig. 10, the wear model needs input information in terms of the initial tool geometry, the tabulated data of the interpolated bouncing back heights, the material
Table 6 List of experimentally evaluated values for the fitting parameters $A_{1}, A_{2}$ and $A_{3}$ for different combinations of tool geometry and fibre cutting angle

\begin{tabular}{llllllllllllll}
\hline Tool geometry & \multicolumn{1}{l}{ E (10/7) } & \multicolumn{4}{c}{ H (10/14) } & \multicolumn{4}{c}{ I (10/21) } & \multicolumn{4}{c}{ L (20/14) } \\
\hline & $A_{1}$ & $A_{2}$ & $A_{3}$ & $A_{1}$ & $A_{2}$ & $A_{3}$ & $A_{1}$ & $A_{2}$ & $A_{3}$ & $A_{1}$ & $A_{2}$ & $A_{3}$ \\
$\Phi=0^{\circ}$ & 0.08 & 0.08 & 0.034 & 0.08 & 0.066 & 0.024 & 0.09 & 0.063 & 0.03 & 0.06 & 0.084 & 0.023 \\
$\Phi=30^{\circ}$ & - & - & - & 0.04 & 0.091 & 0.07 & - & - & - & - & - & - \\
$\Phi=60^{\circ}$ & - & - & - & 0.07 & 0.105 & 0.1 & - & - & - & - & - & - \\
$\Phi=90^{\circ}$ & - & - & - & 0.14 & 0.128 & 0.055 & - & - & - & - & - & - \\
\hline
\end{tabular}


and process properties required for Voss' analytical force model and the values of the fitting parameters $A_{1}, A_{2}$ and $A_{3}$. The values of $A_{1}, A_{2}$ and $A_{3}$ have to be determined for each individual set of the initial rake angle, the initial clearance angle and the fibre cutting angle but are not functions of the cutting length. For their orthogonal machining experiments, Seeholzer et al. [20] used the identical CFRP material, tool substrate, process parameters and test rig configuration as previously used by Voss et al. [8] for their model validation. Consequently, the material and process properties required for Voss' analytical force model are identical to those of the original publication. For the sake of completeness, they are summarised in Table 5.

In a first step, the information about the initial tool geometry and the fibre cutting angle is used to calculate the theoretical bouncing back height at $l_{c u t}=0 \mathrm{~m}$ by extrapolating the trend functions based on the measured points in Table 4 . In a second step, the calculated value of $b_{c}$ is used to localise the contact points $A, B, C$ and $D$ and to calculate the corresponding arc length according to Eq. (3). As a result, the initial active micro-geometry $W_{i}$ is known and the missing wear parameters $\gamma^{*}, \alpha^{*}, l_{\gamma}$ and $l_{\alpha}$ can be calculated accordingly. Since it is the tool's initial state, these parameters have to be identical to $\gamma, \alpha$ and $r_{\text {peak }}$ as mentioned before.

Once the initial active micro-geometry is quantified in terms of $\gamma^{*}, \alpha^{*}, l_{\gamma}, l_{\alpha}$ and $b_{c}$, the region-specific cutting and thrust force components can be calculated using Voss' analytical force model. Based on those, the WRD associated to $W_{0}$ can be determined according to the procedure explained in Sect. 4. In a next step, the calculated WRD is used to update the actual tool shape of $W_{0}$ by using the concept of wear vectors reversely. In combination with the tabulated bouncing back data, this allows the determination of the updated active micro-geometry $W_{1}$. For this updated cutting edge geometry $W_{1}$, the contact points $A$, $B, C$ and $D$ have to be identified anew which then, in combination with the new tool shape, allows the determination of the updated wear parameters $\gamma^{*}, \alpha^{*}, l_{\gamma}$ and $l_{\alpha}$. Subsequently, the value of the arc length parameter is updated with respect to the cutting length increment $\Delta l_{\text {cut }}$ used for the WRD. For the wear simulation in this work, a cutting length increment is varied for different cutting lengths. For cutting lengths between $l_{\text {cut }}=0 \mathrm{~m}$ and $l_{\text {cut }}=5 \mathrm{~m}$, a (a) Overlay of cutting edge profiles

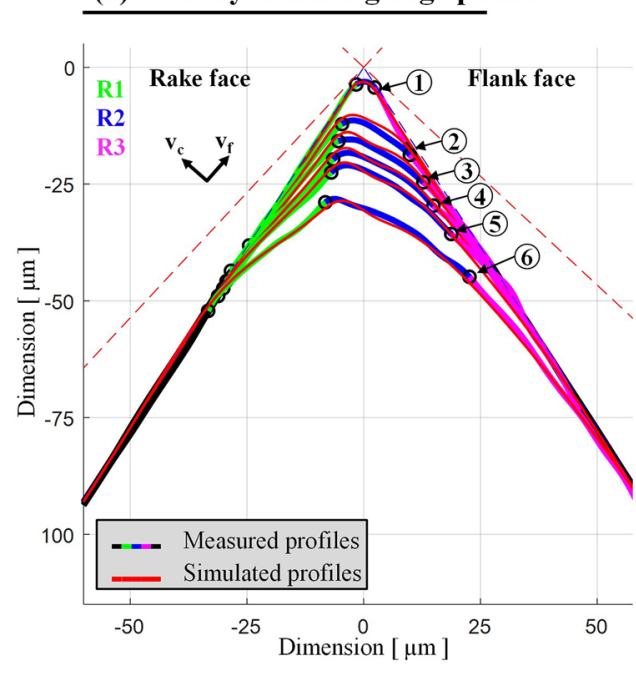

(1) $\mathrm{I}_{\text {cut }}=0 \mathrm{~m}$ (4) $\mathrm{I}_{\text {cut }}=15 \mathrm{~m}$ Tool: $\mathbf{H}(\mathbf{1 0} / \mathbf{1 4})$

(2) $\mathrm{I}_{\text {cut }}=5 \mathrm{~m}$ (5) $\mathrm{I}_{\text {cut }}=20 \mathrm{~m}$ Fibre cutting angle: $\boldsymbol{\Phi}=\mathbf{0}^{\circ}$

(3) $\mathrm{I}_{\text {cut }}=10 \mathrm{~m}$ (6) $\mathrm{I}_{\text {cut }}=35 \mathrm{~m}: \mathrm{v}_{\mathrm{c}}=90 \mathrm{~m} / \mathrm{min}$

\section{(b) Worn tool material}

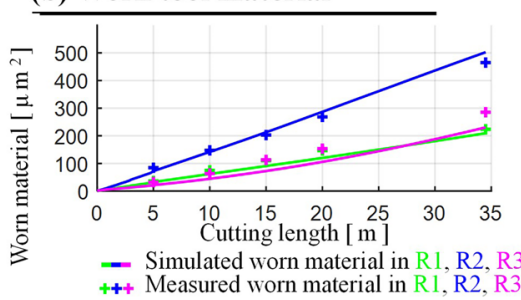

(c) Cutting force

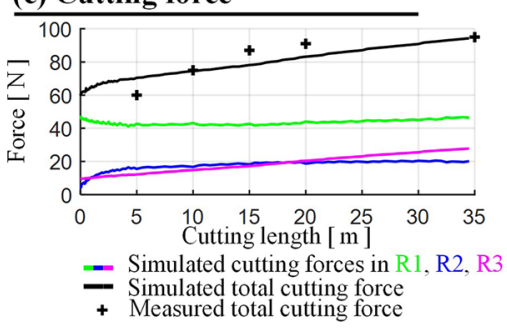

(d) Thrust force

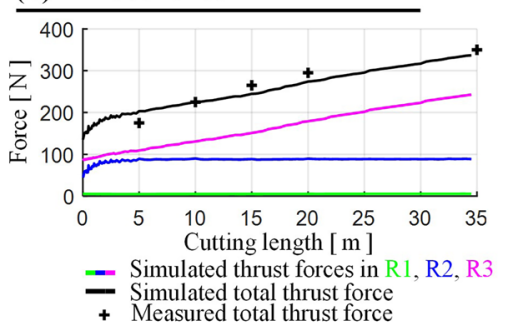

(e) Wear rate distributions
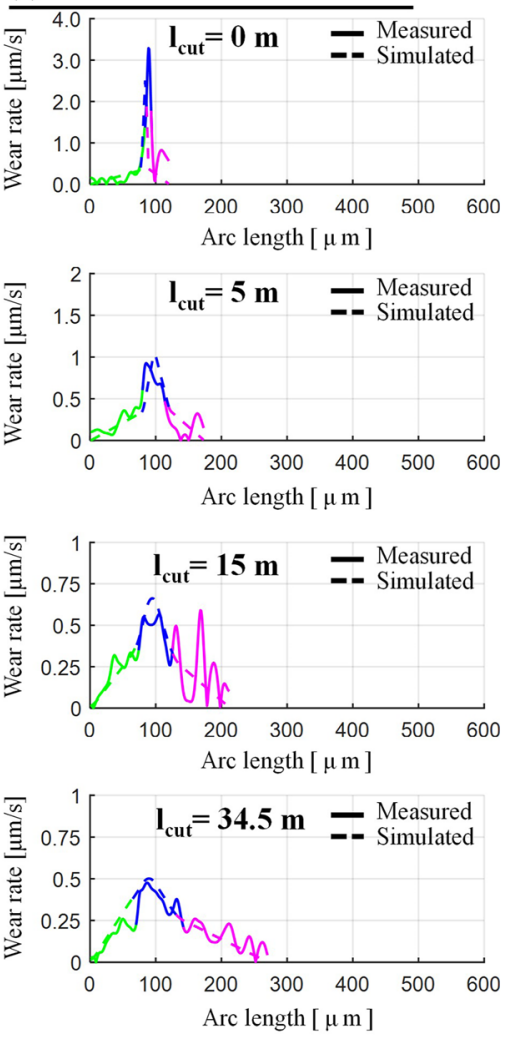

Fig. 11 Comparison of measured and simulated tool wear progression in terms of the cutting edge profiles (a), the amount of worn tool material (b), the trends of cutting and thrust forces $(\mathbf{c}-\mathbf{d})$ and the WRD (e) for tool $\mathrm{H}(10 / 14)$ in machining $\Phi=0^{\circ}\left(v_{c}=90 \mathrm{~m} / \mathrm{min}\right.$, $f=0.03 \mathrm{~mm}, \Delta l_{\text {cut }}=0.05 \mathrm{~m} / 0.5 \mathrm{~m}, A_{1}=0.08, A_{2}=0.066, A_{3}=0.024$ ) 
(a) Overlay of cutting edge profiles

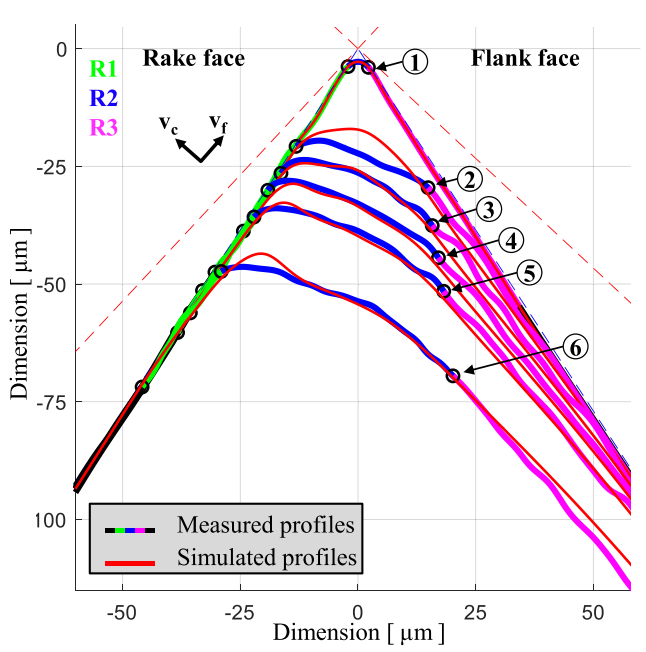

(1) $\mathrm{l}_{\text {cut }}=0 \mathrm{~m}$ (4) $\mathrm{l}_{\text {cut }}=15 \mathrm{~m}$ Tool: $\mathbf{H}(\mathbf{1 0} / \mathbf{1 4})$

(2) $1_{\text {cut }}=5 \mathrm{~m}$ (5) $\mathrm{l}_{\text {cut }}=20 \mathrm{~m}$ Fibre cutting angle: $\Phi=30^{\circ}$

(3) $1_{\text {cut }}=10 \mathrm{~m}$ (6) $1_{\text {cut }}=35 \mathrm{~m}: \mathrm{v}_{\mathrm{c}}=90 \mathrm{~m} / \mathrm{min}$ (b) Worn tool material

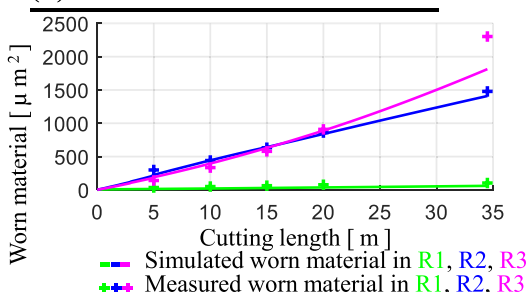

(c) Cutting force

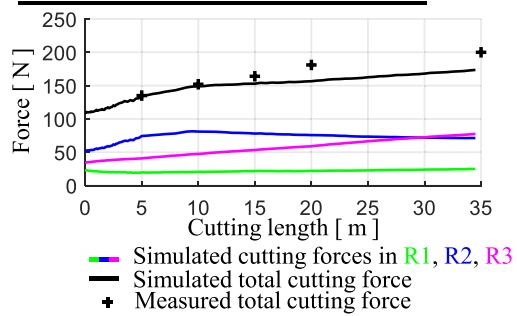

(d) Thrust force

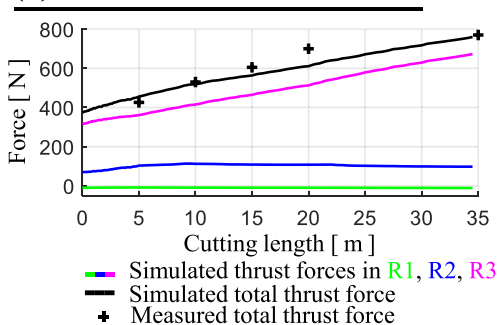

(e) Wear rate distributions
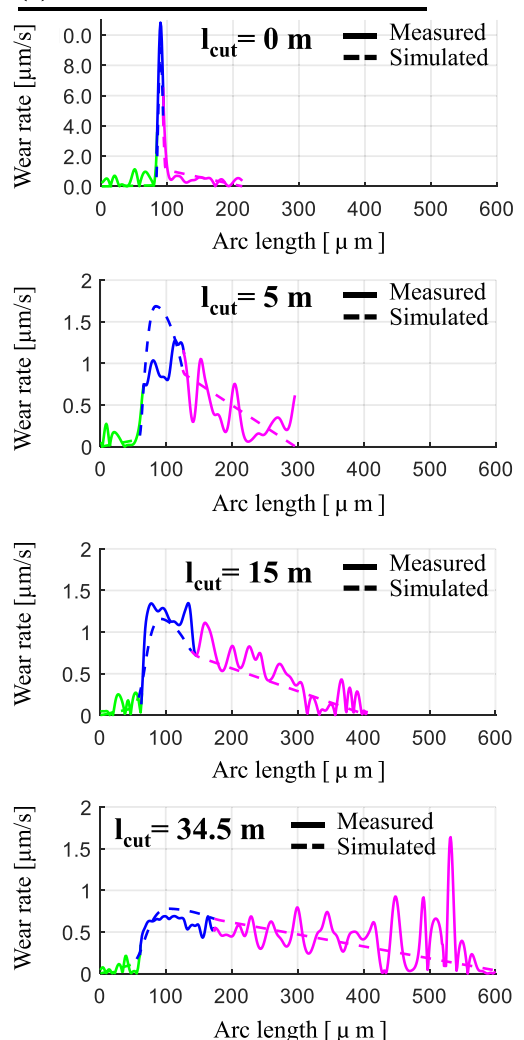

Fig. 12 Comparison of measured and simulated tool wear progression in terms of the cutting edge profiles (a), the amount of worn tool material (b), the trends of cutting and thrust forces $(\mathbf{c}-\mathbf{d})$ and the

cutting length increment of $\Delta l_{\text {cut }}=0.05 \mathrm{~m}$ is used. For cutting lengths $l_{\text {cut }}>5 \mathrm{~m}$, a larger cutting length increment of $\Delta l_{\text {cut }}=0.5 \mathrm{~m}$ is used in order to reduce the simulation time. If the maximum cutting length of $l_{c u t}=35 \mathrm{~m}$ is reached, the wear simulation is completed; otherwise, the abovementioned steps are repeated loop wise according to the schematic illustration in Fig. 10.

An effective way to determine the values of the fitting parameters $A_{1}, A_{2}$ and $A_{3}$ is to compare the experimental and the simulated shapes of the active micro-geometry in R1, R2 and R3 during an entire orthogonal machining process. Subsequently, parameter fitting to the experiments is used to determine one single value for $A_{1}, A_{2}$ and $A_{3}$ that each minimises the error between the experimental and simulated shapes for all cutting length increments simultaneously. The values of the fitting parameters $A_{1}, A_{2}$ and $A_{3}$ have to be evaluated for each tested set of the initial tool geometry and the fibre cutting angle. A list of the evaluated values for all tested combinations of $\gamma, \alpha$ and $\Phi$ is shown in Table 6.
WRD (e) for tool $\mathrm{H}(10 / 14)$ in machining $\Phi=30^{\circ}\left(v_{c}=90 \mathrm{~m} / \mathrm{min}\right.$, $f=0.03 \mathrm{~mm}, \Delta l_{\text {cut }}=0.05 \mathrm{~m} / 0.5 \mathrm{~m}, A_{1}=0.04, A_{2}=0.091, A_{3}=0.07$ )

\section{Simulation results and model validation}

For validation purposes, the simulated tool wear progression and the corresponding trends of cutting and thrust forces are compared to the experimental results. In accordance with Sect. 5, the values of $n_{w}$ in Table 3, the tabulated bouncing back heights in Table 4, the material and process parameters in Table 5, and the experimentally evaluated values of $A_{1}, A_{2}$ and $A_{3}$ in Table 6 are used as inputs for the simulation.

The model validation is divided into four categories in order to verify different quality criteria of the wear modelling approach. These four validation categories are as follows:

- Comparing the shape of the active micro-geometry

- Comparing the region-specific amount of worn tool material

- Comparing the WRD

- Comparing the cutting and thrust forces 
Comparing the simulated and measured process forces is not to validate Voss's analytical force model but to prove its compatibility with the wear modelling approach introduced in this paper. As explained in Sect. 5, the force model uses the five wear parameters $\gamma^{*}, \alpha^{*}, l_{\gamma}, l_{\alpha}$ and $b_{c}$ as input data in order to approximate the shape of the active micro-geometry and subsequently uses this information for region-specific cutting and thrust force determination. In contrast to Voss's original paper, in this work, the wear parameters $\gamma^{*}, \alpha^{*}, l_{\alpha}$ and $l_{\gamma}$ are not provided as a look-up table but are calculated anew in each iteration during the wear simulation procedure according to Fig. 10. During the simulation, small errors in the simulated tool shapes or in localising the contact points $A, B, C$ and $D$ would lead to incorrect values for $\gamma^{*}, \alpha^{*}, l_{\gamma}, l_{\alpha}$ and hence wrong cutting and thrust forces. Subsequently, this would lead to incorrect mean wear rates and thus not representative WRD. Therefore, the error would accumulate over the duration of the simulation which means that the tool's wear progression cannot be simulated reasonably.

According to Sect. 3.2.2, the WRD and its change with progressive tool wear clearly depend on the initial tool geometry and the fibre cutting angle which results in different wear progressions. To consider both influencing factors separately, the model validation in terms of the four mentioned validation categories is done in two steps. First in Sect. 6.1, the model validation is done in terms of the fibre cutting angle. For this purpose, the simulated and experimentaly determined results are compared for the tool $\mathrm{H}(10 / 14)$ in machining $\Phi=0^{\circ}, \Phi=30^{\circ}, \Phi=60^{\circ}$ and $\Phi=90^{\circ}$. Second in Sect. 6.2, the model validation is done in terms of the initial tool geometry. This is representatively done for machining $\Phi=0^{\circ}$, where the four different tool geometries E (10/7), H (10/14), I (10/21) and L (20/14) are considered. Comparing the simulated and experimentally determined results for the tools E (10/7), H (10/14) and I (10/21) gives information about the wear model's capability to reproduce the influence of the initial clearance angle on tool wear. Similarly, comparing the tools H (10/14) and L (20/14) gives information about the wear model's capability to reproduce the influence of the initial rake angle on tool wear.

In general, all wear simulations are started from the measured initial tool shape at $l_{\text {cut }}=0 \mathrm{~m}$. The only exception is (a) Overlay of cutting edge profiles

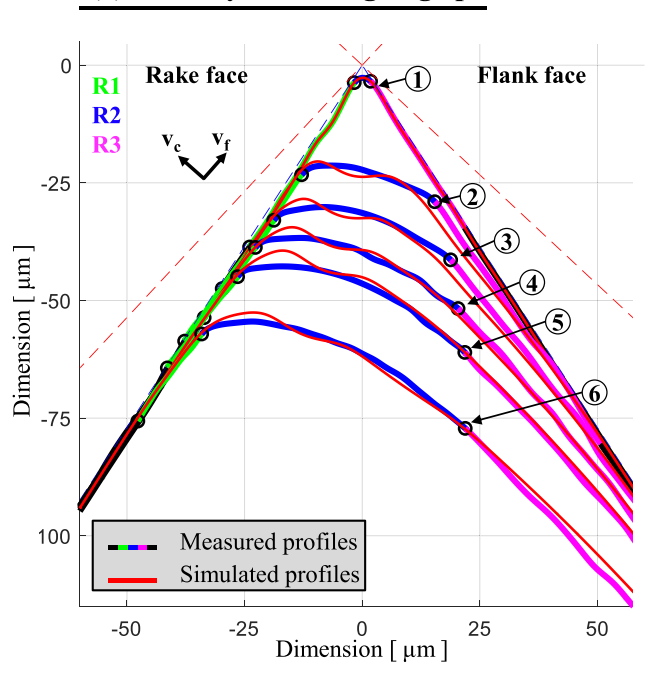

\begin{tabular}{ll|l|l}
\hline (1) $1_{\text {cut }}=0 \mathrm{~m}$ & (4) $1_{\text {cut }}=15 \mathrm{~m}$ & Tool: $\mathbf{H}(\mathbf{1 0} / \mathbf{1 4})$
\end{tabular}

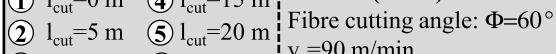

(3) $\mathrm{I}_{\text {cut }}=10 \mathrm{~m}$ (6) $\mathrm{I}_{\text {cut }}=35 \mathrm{~m}: \begin{aligned} & \mathrm{v}_{\mathrm{c}}=90 \mathrm{~m} / \mathrm{min} \\ & \mathrm{f}=0.03 \mathrm{~mm} / \mathrm{rev}\end{aligned}$

\section{(b) Worn tool material}

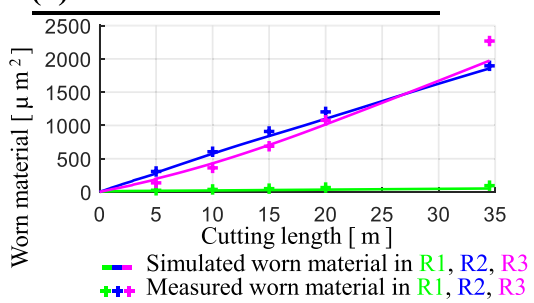

(c) Cutting force

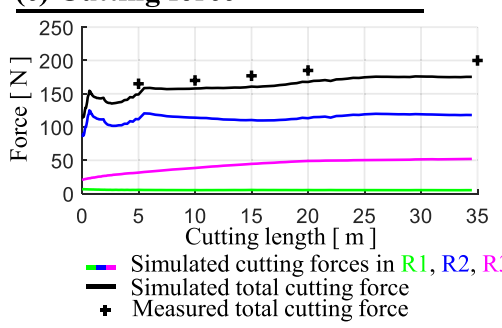

(d) Thrust force

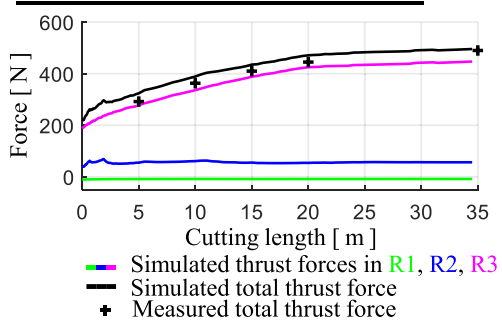

\section{(e) Wear rate distributions}
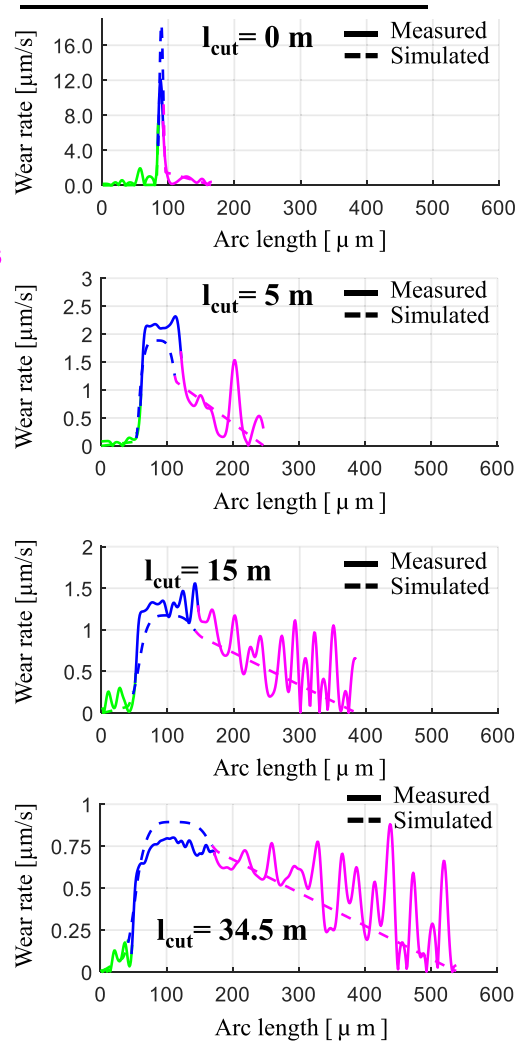

Fig. 13 Comparison of measured and simulated tool wear progression in terms of the cutting edge profiles (a), the amount of worn tool material (b), the trends of cutting and thrust forces $(\mathbf{c}-\mathbf{d})$ and the
WRD (e) for tool $\mathrm{H}(10 / 14)$ in machining $\Phi=60^{\circ}\left(v_{c}=90 \mathrm{~m} / \mathrm{min}\right.$, $f=0.03 \mathrm{~mm}, \Delta l_{\text {cut }}=0.05 \mathrm{~m} / 0.5 \mathrm{~m}, A_{1}=0.07, A_{2}=0.105, A_{3}=0.1$ ) 
the tool $\mathrm{H}(10 / 14)$ in machining $\Phi=90^{\circ}$, which is started from the measured tool shape at $l_{\text {cut }}=5 \mathrm{~m}$ due to an early incompatibility of the wear and force models as explained in detail in Sect. 6.1.4.

\subsection{Model validation in terms of the fibre cutting angle}

\subsubsection{Validation - tool $\mathrm{H}(10 / 14)-\Phi=0^{\circ}$}

Figure 11 depicts the validation overview for tool $\mathrm{H}(10 / 14)$ in machining $\Phi=0^{\circ}$ which is separated into eight subareas. These areas are explained in the following and are representative for the remaining validation overviews in the subsequent sections.

Figure 11a shows the overlay of measured and simulated cutting edge profiles at the cutting lengths of $l_{c u t}=0 \mathrm{~m}$, $l_{\text {cut }}=5 \mathrm{~m}, l_{\text {cut }}=10 \mathrm{~m}, l_{\text {cut }}=15 \mathrm{~m}, l_{\text {cut }}=20 \mathrm{~m}$ and $l_{\text {cut }}=35 \mathrm{~m}$. For this purpose, the simulated profiles are shown in red while the measured profiles are shown in green, blue and magenta with respect to the three regions R1, R2 and R3. Figure $11 \mathrm{~b}$ reveals the comparison of the simulated and measured results for the worn tool material as function of the cutting length. In this context, the measured data is depicted as single points with respect to the five analysis steps during the orthogonal machining experiments explained in Sect. 3 . The simulated data is presented as trend lines based on the applied cutting length increment. Figure 11c shows the comparison of the experimentally and analytically determined cutting forces, where the measured force data is depicted as single points with respect to the five analysing steps. The simulated results are shown by means of four different curves. The black line represents the total simulated cutting force and the green, blue and magenta lines represent the corresponding region-specific cutting force components. The sum of all region-specific cutting force components is equal to the total cutting force. Analogous to Fig. 11c, Fig. 11d shows the comparison of the experimentally and analytically determined thrust forces. Finally, Fig. 11e enables the comparison of the simulated and measured WRD which are representatively shown for the cutting lengths of $l_{\text {cut }}=0 \mathrm{~m}, l_{\text {cut }}=5 \mathrm{~m}$, $l_{c u t}=15 \mathrm{~m}$ and $l_{\text {cut }}=34.5 \mathrm{~m}$. Using the same colour notation as in Fig. 11a, the measured and simulated WRD are shown as solid and dashed lines, respectively. (a) Overlay of cutting edge profiles

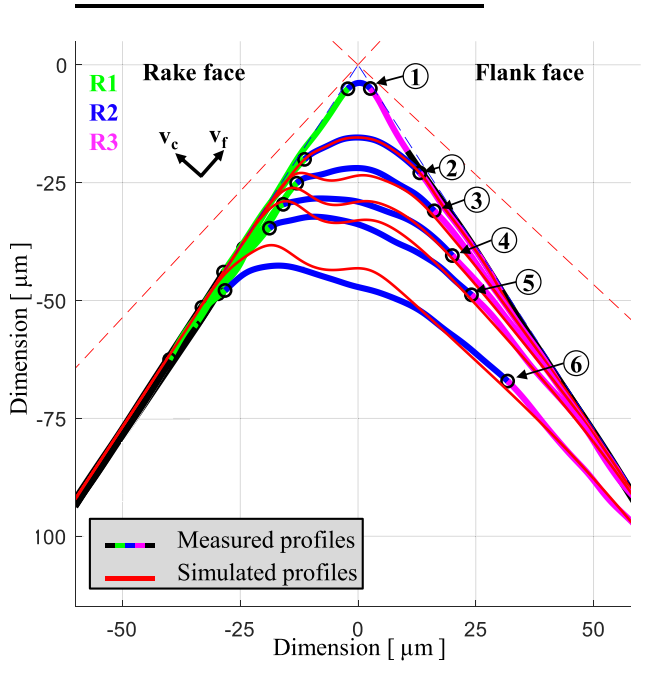

\begin{tabular}{ll|l} 
(1) $1_{\text {cut }}=0 \mathrm{~m}$ & (4) $l_{\text {cut }}=15 \mathrm{~m}$ & Tool: $\mathbf{H}(\mathbf{1 0} / \mathbf{1 4})$
\end{tabular}

(2) $1_{\text {cut }}=5 \mathrm{~m}$ (5) $1_{\text {cut }}=20 \mathrm{~m}$ Fibre cutting angle: $\Phi=90^{\circ}$

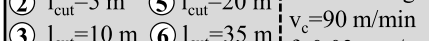

(b) Worn tool material

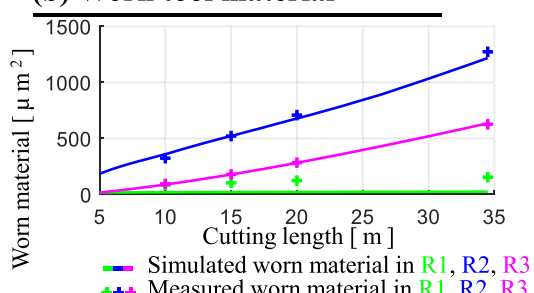

(c) Cutting force

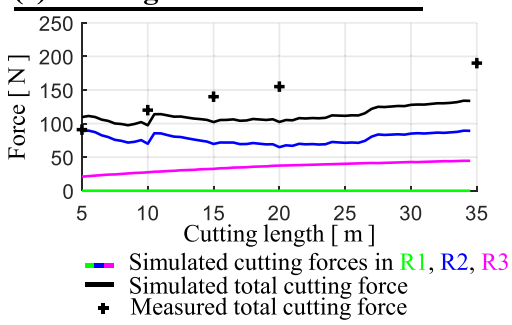

(d) Thrust force

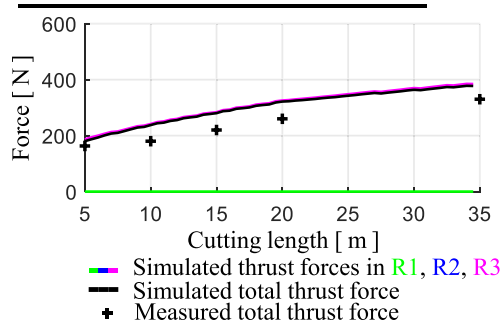

(e) Wear rate distributions
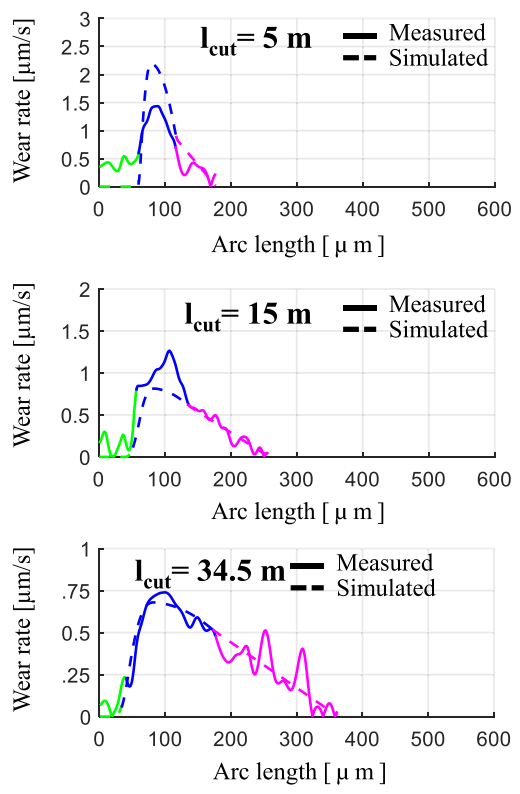

Fig. 14 Comparison of measured and simulated tool wear progression in terms of the cutting edge profiles (a), the amount of worn tool material (b), the trends of cutting and thrust forces $(\mathbf{c}-\mathbf{d})$ and the
WRD (e) for tool $\mathrm{H}(10 / 14)$ in machining $\Phi=90^{\circ}\left(v_{c}=90 \mathrm{~m} / \mathrm{min}\right.$, $f=0.03 \mathrm{~mm}, \Delta l_{\text {cut }}=0.05 \mathrm{~m} / 0.5 \mathrm{~m}, A_{1}=0.14, A_{2}=0.128, A_{3}=0.055$ ) 
As shown in Fig. 11e, the simulated and measured WRD are in good agreement for all presented cutting lengths. Accordingly, the wear model is capable of reproduce the WRD's shape and magnitude as well as its region-specific segmentation into R1, R2 and R3. Furthermore, it can be seen that for most cutting lengths, the chosen representative position of $W_{\max }$ by means of the experimentally determined value of $n_{w}$ is a good approximation to reality. The noticeable amount of fluctuation in R3, as particularly seen for $l_{\text {cut }}=15 \mathrm{~m}$, is explained by occurring misalignments during the profile interpolation as discussed in detail in Sect. 3.2.1. According to Fig. 11a, only small deviations between the simulated and measured cutting edge profiles can be identified which means that not only the selected WRD in Fig. 11e but also the continuous change of the WRD with increasing cutting length can be approximated well with the proposed wear modelling approach. Moreover, the wear model is capable to reproduce the most important wear characteristics for the tool $\mathrm{H}(10 / 14)$ in machining $\Phi=0^{\circ}$ that are the intense tool wear in $\mathrm{R} 1$, the asymmetric cutting edge rounding in $\mathrm{R} 2$, the decreasing clearance angle in $\mathrm{R} 3$ and the simultaneously increasing friction length on the flank face.
In agreement with the realistic wear progression seen in Fig. $11 \mathrm{a}, \mathrm{b}$ reveals that the region-specific amount of worn tool material and its change with increasing cutting length can be reproduced well with the wear model. Accordingly, the most intense material loss is found in R3, followed by R1 and R2 that are comparable. Occurring deviations between the simulated and measured material loss are particularly attributed to the simplified shape parameterisation approach and the concept of mean wear rates.

As shown in Fig. 11c, d, not only the tool wear progression but also the corresponding changes in cutting and thrust forces with increasing cutting length can be approximated realistically. Occurring deviations can be particularly found in the tool's early wear state and can be attributed to the simplifications of the force and wear models. The largest differences between the simulated and measured data are found for $l_{\text {cut }}=5 \mathrm{~m}$, where the total cutting and thrust forces are overestimated by $18 \%$ and $17.5 \%$, respectively. Despite this, the characteristic rises of cutting and thrust forces with progressive tool wear can be reproduced well. According to Fig. 11c, the simulated cutting force for $l_{c u t}<5 \mathrm{~m}$ is dominated by the force contribution of $\mathrm{R} 1$. With increasing (a) Overlay of cutting edge profiles

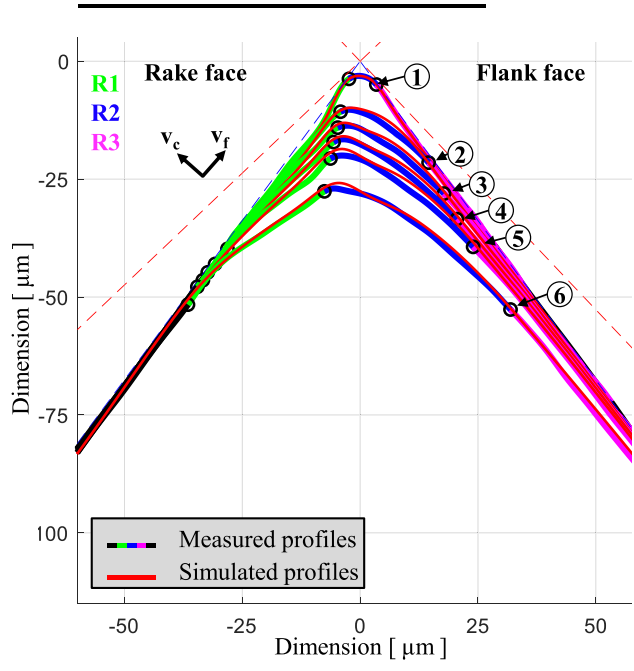

\begin{tabular}{ll|l} 
(1) $1_{\text {cut }}=0 \mathrm{~m}$ & (4) $1_{\text {cut }}=15 \mathrm{~m}$ & Tool: $\mathbf{E}(\mathbf{1 0} / 7)$
\end{tabular}

(2) $1_{\text {cut }}=5 \mathrm{~m}$ (5) $1_{\text {cut }}=20 \mathrm{~m}$ Fibre cutting angle: $\Phi=0^{\circ}$

(3) $1_{\text {cut }}=10 \mathrm{~m}$ (6) $1_{\text {cut }}=35 \mathrm{~m}: \mathrm{v}_{\mathrm{c}}=90 \mathrm{~m} / \mathrm{min}$ (b) Worn tool material

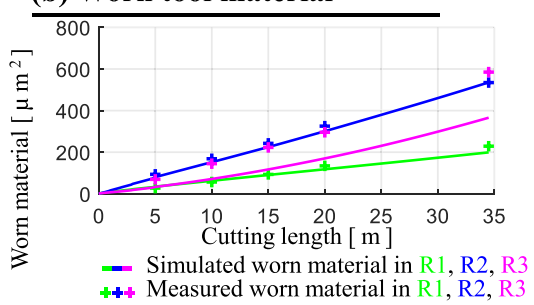

(c) Cutting force

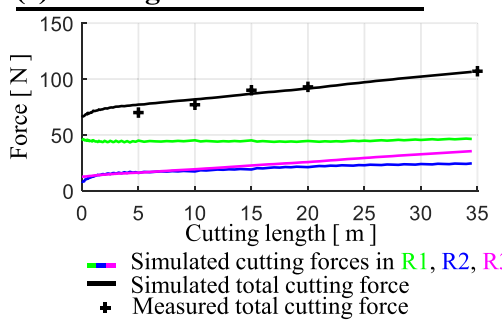

(d) Thrust force

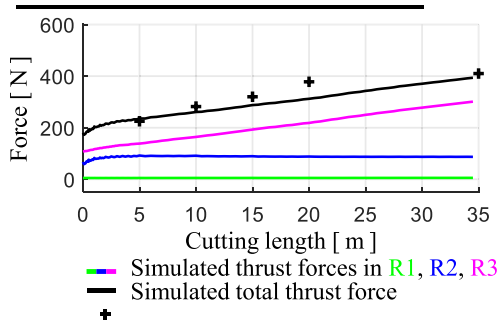

(e) Wear rate distributions
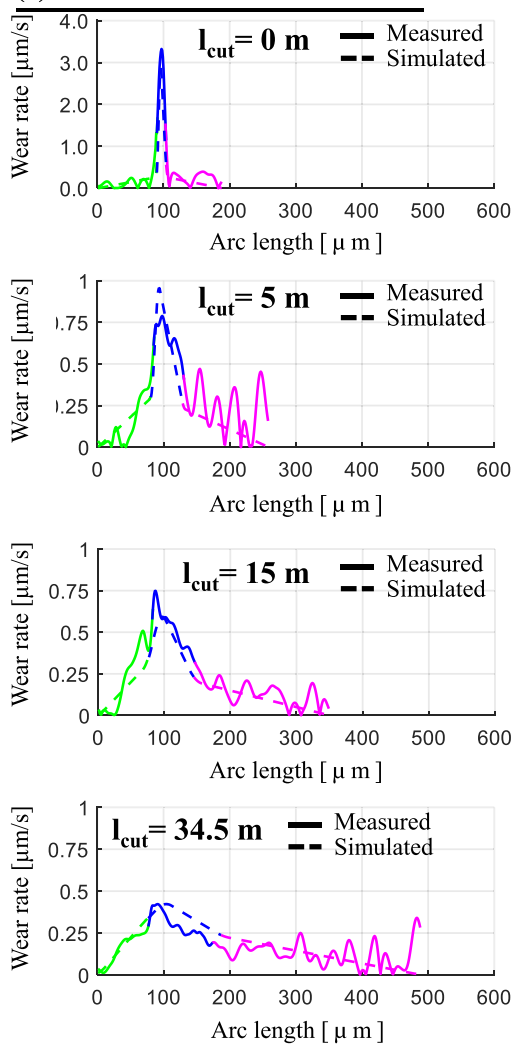

Fig. 15 Comparison of measured and simulated tool wear progression in terms of the shape of the cutting edge (a), the amount of worn tool material (b), the trends of cutting and thrust forces $(\mathbf{c}-\mathbf{d})$ and the WRD (e) for tool E (10/7) in machining $\Phi=0^{\circ}\left(v_{c}=90 \mathrm{~m} / \mathrm{min}\right.$, $f=0.03 \mathrm{~mm}, \Delta l_{\text {cut }}=0.05 \mathrm{~m} / 0.5 \mathrm{~m}, A_{1}=0.08, A_{2}=0.08, A_{3}=0.034$ ) 
cutting length, in particular, the contribution of R3 to the overall cutting force increases clearly, which can be attributed to the substantial flank wear seen in Fig. 11a. As shown in Fig. 11d, the simulated thrust force is dominated by the force components in R2 and R3, whereas the latter increases in importance with increasing cutting length due to the substantial flank wear mentioned before.

\subsubsection{Validation - tool $H(10 / 14)-\Phi=30^{\circ}$}

Analogous to the presentation style of Figs. 11 and 12 depicts the validation overview for the tool $\mathrm{H}(10 / 14)$ in machining $\Phi=30^{\circ}$. As shown in Fig. 12e, the simulated and measured WRD are in good agreement for most of the presented cutting lengths while the chosen representative position of $W_{\max }$ is a good first approximation. By comparing Figs. 11e and 12e, it can be seen that the wear model is capable to reproduce the influence of the changed fibre cutting angle from $\Phi=0^{\circ}$ to $\Phi=30^{\circ}$ on the wear distribution's shape and size. The most significant difference between the measured and simulated WRD is found in the relatively new state of the cutting tool at $l_{\text {cut }}=5 \mathrm{~m}$, where the tool wear in R2 is underestimated.
The consequence of this underestimation can be seen in the simulated cutting edge profiles in Fig. 12a, where the simulated shape in $\mathrm{R} 2$ does not agree well with the measured results.

In direct comparison of the measured WRD at $l_{\text {cut }}=5 \mathrm{~m}$ to those at $l_{\text {cut }}=0 \mathrm{~m}, l_{\text {cut }}=15 \mathrm{~m}$ and $l_{\text {cut }}=34.5 \mathrm{~m}$, it can be seen that in particular, R2 has an unusual shape with a local minimum in the middle. It is assumed that part of the tool in R2 broke off shortly before the measurement which coincides with the straight shape of the measured cutting edge profile seen in Fig. 12a. As chipping is not considered in the modelling approach, the wear model is not capable to reproduce this instant change in tool shape accurately. However, chipping seems to be the exception as it is only found for this tool geometry and this fibre cutting angle. Moreover, the risk for chipping seems to be larger in the tool's early wear state since for all subsequent wear states, no chipping is identified as shown in Fig. 12a. Beside chipping, the considered simplifications of the wear and force models (the shape parameterisation of the WRD, the simplification of the micro-geometry to the five wear parameters, usage of mean wear rates, etc.) and numerical issues during the simulation, such as (a) Overlay of cutting edge profiles

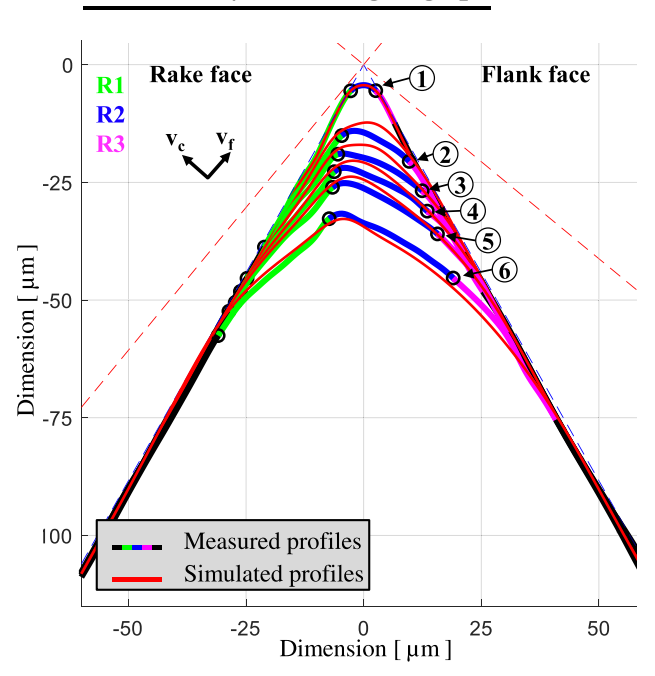

\begin{tabular}{ll|l} 
(1) $l_{\text {cut }}=0 \mathrm{~m}$ & (4) $l_{\text {cut }}=15 \mathrm{~m}$ & Tool: $\mathbf{I}(\mathbf{1 0} / \mathbf{2 1})$
\end{tabular}

(2) $\mathrm{I}_{\text {cut }}=5 \mathrm{~m}$ (5) $\mathrm{I}_{\mathrm{cut}}=20 \mathrm{~m}:$ Fibre cutting angle: $\Phi=0^{\circ}$

(3) $1_{\text {cut }}=10 \mathrm{~m}$ (6) $1_{\mathrm{cut}}=35 \mathrm{~m}: \mathrm{v}_{\mathrm{c}}=90 \mathrm{~m} / \mathrm{min}$ (b) Worn tool material

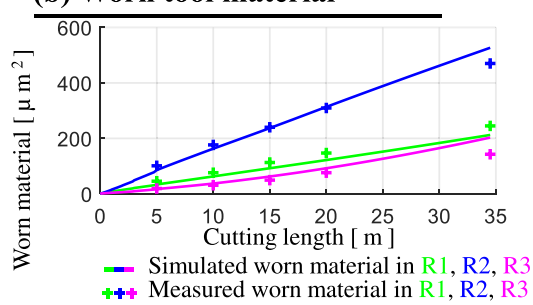

\section{(c) Cutting force}

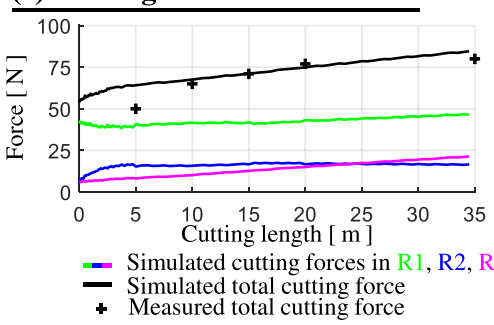

(d) Thrust force

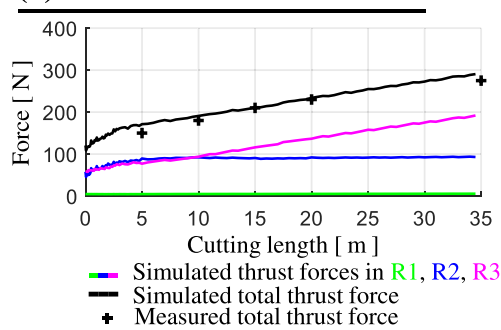

(e) Wear rate distributions
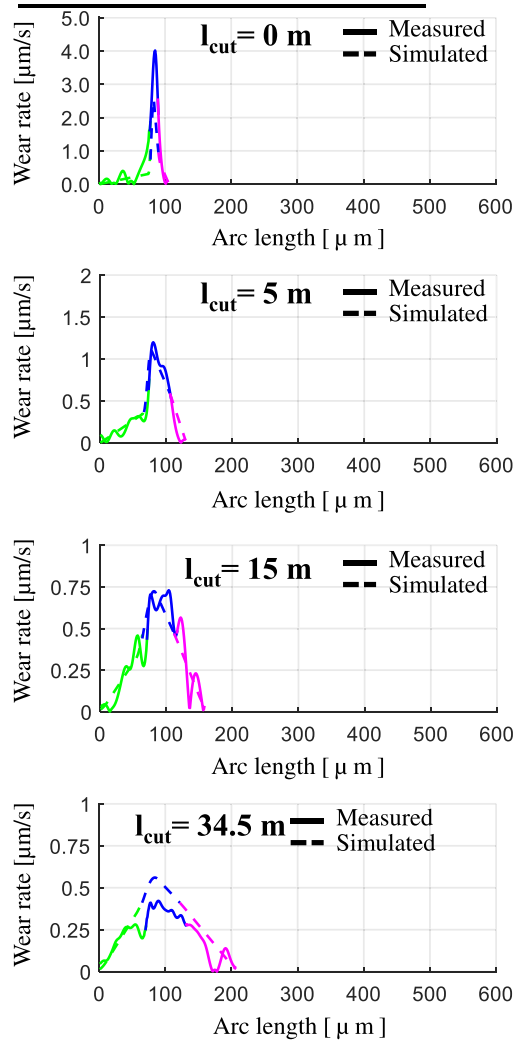

Fig. 16 Comparison of measured and simulated tool wear progression in terms of the shape of the cutting edge (a), the amount of worn tool material (b), the trends of cutting and thrust forces $(\mathbf{c}-\mathbf{d})$ and the WRD (e) for tool I (10/121) in machining $\Phi=0^{\circ}\left(v_{c}=90 \mathrm{~m} / \mathrm{min}\right.$, $f=0.03 \mathrm{~mm}, \Delta l_{\text {cut }}=0.05 \mathrm{~m} / 0.5 \mathrm{~m}, A_{1}=0.09, A_{2}=0.063, A_{3}=0.03$ ) 
polynomial overshoots during the shape approximation, can be responsible for the differences between simulation and experiment. A possible counteractive method for reducing the overshooting could be the application of a smoothing filter after each simulation step. However, in order to reduce the significance of external influencing factors on the simulation results and to avoid overfitting, no filter options are considered in this work.

For cutting length $l_{\text {cut }}>5 \mathrm{~m}$, the wear model is capable to reproduce the tool wear progression realistically. The most important wear characteristics for the tool $\mathrm{H}(10 / 14)$ in machining $\Phi=30^{\circ}$ can be simulated which includes the almost negligible tool wear in R1, the increasing asymmetric cutting edge rounding in R2, the continuously decreasing clearance angle in R3 and the simultaneously increasing arc length.

According to Fig. 12b, the simulated material losses in $\mathrm{R} 1, \mathrm{R} 2$ and $\mathrm{R} 3$ are in good agreement with the experimental data. While the removed tool material in R1 is close to zero due to the negligible wear on the rake face, the material losses in R2 and R3 are comparable and increase clearly with increasing cutting length.
The comparison of experimentally and analytically determined cutting and thrust forces is revealed in Fig. 12c, d. For both force components, the simulation is in good agreement with the experiment. The largest differences between the simulated and measured data are found for $l_{\text {cut }}=20 \mathrm{~m}$, where the total cutting and thrust forces are underestimated by $15 \%$ and $14.5 \%$, respectively. In particular, the thrust force is dominated by the force contribution of R3 which in accordance with Fig. 12a, e is attributed to intense flank wear resulting in a long total arc length.

\subsubsection{Validation - tool $H(10 / 14)-\Phi=60^{\circ}$}

Figure 13 shows the validation overview for the tool geometry $\mathrm{H}$ (10/14) in machining $\Phi=60^{\circ}$. According to Fig. 13e, the WRD and its change with progressive tool wear can be approximated well with the wear model which includes the decreasing peak value and the simultaneously increasing arc length. As the tool wear progression for $\Phi=60^{\circ}$ is different to those found for $\Phi=0^{\circ}$ and $\Phi=30^{\circ}$, the wear model is capable to reproduce the effect of the fibre cutting angle. Although the simulation is likely to over- or underestimate the wear rate at some point, Fig. 13a reveals only small differences between the experimentally and (a) Overlay of cutting edge profiles

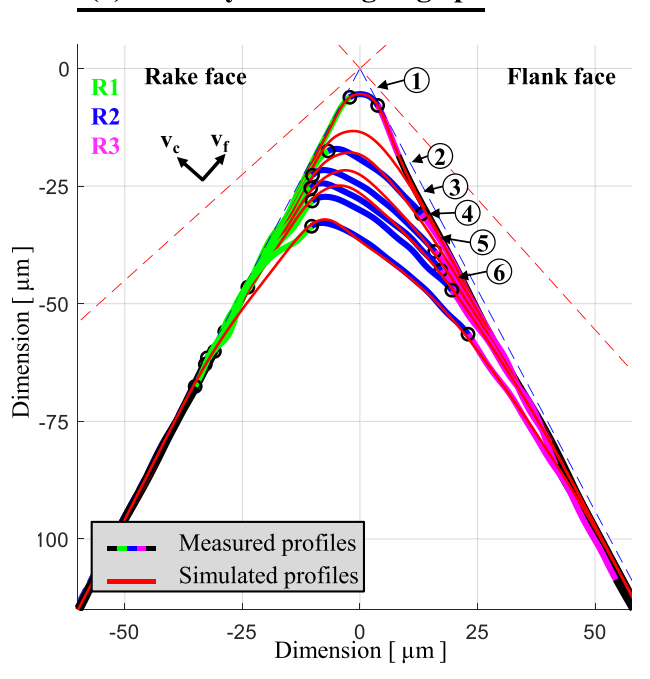

\begin{tabular}{ll|l|l} 
(1) $1_{\text {cut }}=0 \mathrm{~m}$ & (4) $1_{\text {cut }}=15 \mathrm{~m}$ & Tool: $\mathrm{L}$ (20/14)
\end{tabular}

(2) $1_{\text {cut }}=5 \mathrm{~m}$ (5) $1_{\text {cut }}=20 \mathrm{~m}:$ Fibre cutting angle: $\Phi=0^{\circ}$

(3) $\mathrm{I}_{\text {cut }}=10 \mathrm{~m}$ (6) $\mathrm{I}_{\text {cut }}=35 \mathrm{~m}: \mathrm{v}_{\mathrm{c}}=90 \mathrm{~m} / \mathrm{min}$ (b) Worn tool material

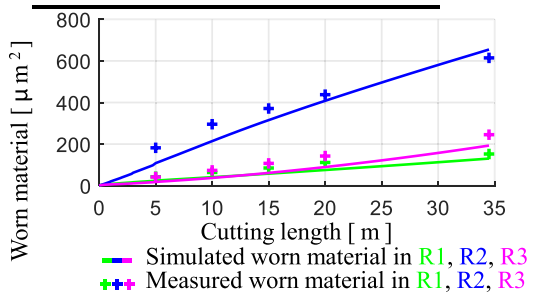

(c) Cutting force

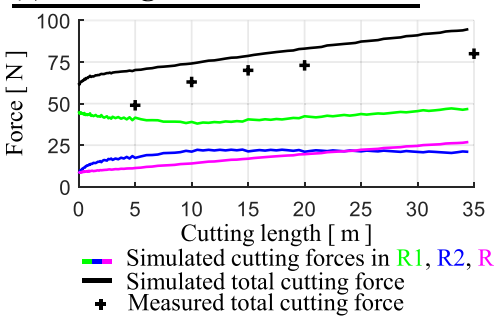

(d) Thrust force

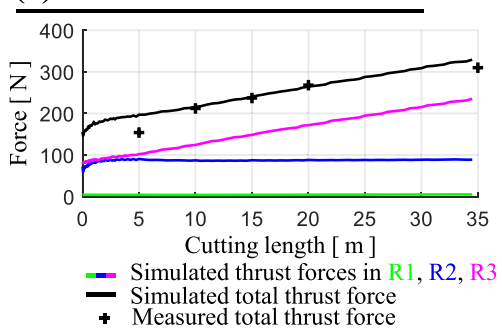

(e) Wear rate distributions
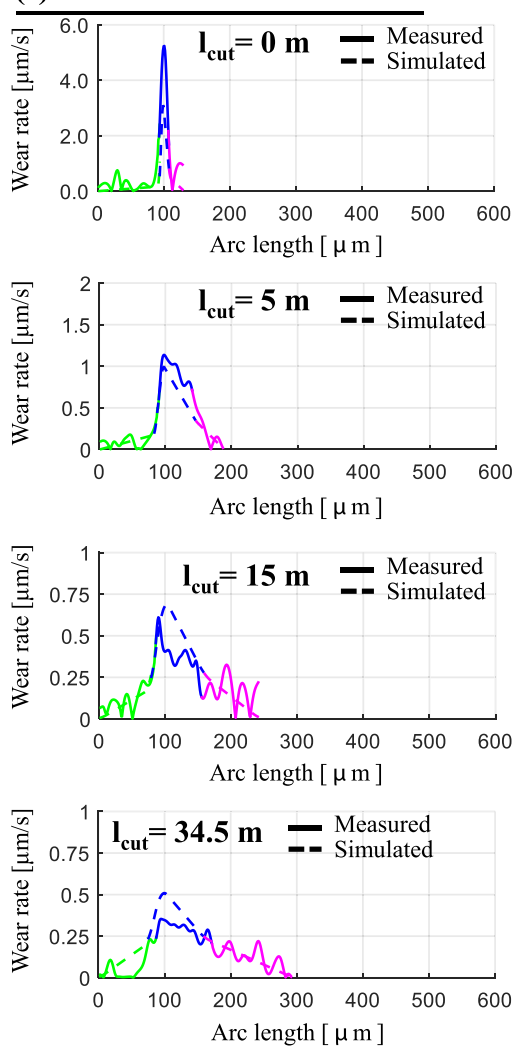

Fig. 17 Comparison of measured and simulated tool wear progression in terms of the shape of the cutting edge (a), the amount of worn tool material (b), the trends of cutting and thrust forces $(\mathbf{c}-\mathbf{d})$ and the WRD (e) for tool L (20/14) in machining $\Phi=0^{\circ}\left(v_{c}=90 \mathrm{~m} / \mathrm{min}\right.$, $f=0.03 \mathrm{~mm}, \Delta l_{\text {cut }}=0.05 \mathrm{~m} / 0.5 \mathrm{~m}, A_{1}=0.06, A_{2}=0.084, A_{3}=0.023$ ) 
analytically determined cutting edge profiles at the five analysing steps. Different to previous findings in Sects. 6.1.1 and 6.1.2, Fig. 13a indicates that in particular, the simulated cutting edge profiles in R2 are characterised by a wavy shape because of an overshooting as already discussed in Sect. 6.1.2. Initially occurring in the early wear state at $l_{c u t}<5 \mathrm{~m}$, this error accumulates during the subsequent simulation loops. As shown in Fig. 13e, the value of $W_{\max }$ of the WRD at $l_{\text {cut }}=5 \mathrm{~m}$ is clearly overestimated with the wear model. During the following updating phase of the cutting edge profile, this overestimation results in an incorrectly distributed material loss in $\mathrm{R} 2$ with a maximum wear rate close to $l_{r_{-} W \max }$. In accordance with Fig. 13e, the simulation's overestimation of $W_{\max }$ can be reduced clearly for the following cutting lengths. This means that the associated material loss is more realistically distributed along R2. However, the distribution error in R2 in the tool's early wear state has already caused an unrealistic tool shape which cannot be corrected during the following updating cycles. Therefore, the wavy shape of R2 can still be seen for the subsequent wear states although the model is capable to reproduce the corresponding WRD. In addition to under- or overestimated WRD, small shape irregularities in the measured initial tool shapes are critical and can intensify the shape distortion.

Separate from deviations in the shape of $\mathrm{R} 2$, the wear model is capable to reproduce the most important wear characteristics which includes the negligible tool wear in R1, the strong asymmetric cutting edge rounding in $\mathrm{R} 2$, the continuously decreasing clearance angle in R3 and the simultaneously increasing arc length. Moreover, Fig. 13b shows that the simulated material losses in R1, R2 and R3 are in good agreement with the experimental data. Comparable to the results in machining $\Phi=30^{\circ}$, the total amount of worn tool material is almost exclusively caused by R2 and R3.

Figure $13 \mathrm{c}$, d show a comparison of the experimentally and analytically determined cutting and thrust forces. The largest difference between the simulated and measured cutting force is found for $l_{\text {cut }}=35 \mathrm{~m}$, where the total cutting force is underestimated by $14 \%$. For the thrust force, with an overestimation of $11 \%$, the largest difference is found for $l_{c u t}=5 \mathrm{~m}$.

\subsubsection{Validation - tool $H(10 / 14)-\Phi=90^{\circ}$}

Figure 14 depicts the validation overview for the tool geometry $\mathrm{H}(10 / 14)$ in machining $\Phi=90^{\circ}$. In contrast to the remaining tool geometries and fibre cutting angles, this simulation is started from the measured cutting edge profile at $l_{\text {cut }}=5 \mathrm{~m}$ instead of the initial tool geometry at $l_{\text {cut }}=0 \mathrm{~m}$. This is because the combination of Voss's force model and the proposed wear modelling approach does not perform well for $l_{\text {cut }}<5 \mathrm{~m}$ in machining $\Phi=90^{\circ}$ with the tool geometry H (20/14). More precisely, the WRD in R2 and its peak value $W_{\max }$ cannot be reproduced well enough for small cutting lengths. During the simulation, this results in wavy tool shapes in R2 as discussed in the section before. In machining $\Phi=90^{\circ}$, these disturbances are large enough that the simulation has to be stopped.

According to Fig. 14, the degree of shape disturbance can be reduced if the simulation is started at $l_{c u t}=5 \mathrm{~m}$ instead of $l_{\text {cut }}=0 \mathrm{~m}$. This is due to the fact that the WRD in R2 can be better reproduced by the modelling approach for $l_{\text {cut }} \leq 5 \mathrm{~m}$ as shown in Fig. 14e. Although the applicability of the wear model is apparently limited for machining $\Phi=90^{\circ}$, the simulated tool shapes in Fig. 14a reflect the most important wear characteristics. Moreover, Fig. 14b reveals that the wear model is capable to reproduce the region-specific material losses in R2 and R3 realistically. The material loss in R1 is slightly underestimated by the simulation.

The experimentally and analytically determined cutting and thrust forces are revealed in Fig. 14c, d. According to Fig. 14c, the cutting force component in R2 does not show a clear trend and is the main reason, why the total cutting force does not follow the trend identified from the experiments. It is assumed that the reason for this is attributed to the wavy shape of R2 which has a negative effect on the prediction accuracy of the force model. The largest deviation for the simulated cutting force is found for $l_{\text {cut }}=35 \mathrm{~m}$, where the total cutting force is underestimated by $41 \%$. As shown in Fig. 14d, the trend of the simulated thrust forces is in good agreement with the measured trend. With an overestimation of $31.6 \%$, the largest difference for the thrust force is found for $l_{\text {cut }}=10 \mathrm{~m}$.

\subsection{Model validation in terms of the initial tool geometry}

Analogous to the model validation in terms of the fibre cutting angle, Figs. 15, 16, and 17 depict the validation overviews for the tools E (10/7), I (10/21) and L (20/14) in machining $\Phi=0^{\circ}$.

In order to validate the wear model's capability to reproduce the influence of the initial clearance angle on tool wear, the simulation results of E (10/7), H (10/14) and I (10/21) in Figs. 11, 15, and 16 have to be compared. For all three tools, the simulation results in terms of the worn tool shape, the WRD, the amount of worn material and the process forces are in good agreement with the experimental data with outliers occurring naturally. Although the simulation tends to over- or underestimate the WRD at some point, the wear model is capable to reproduce the effect of the initial clearance angles on the tool wear progression realistically. For example, it can be simulated that reducing the initial clearance angle from $\alpha=21^{\circ}$ (tool I) to $\alpha=14^{\circ}$ (tool H) and $\alpha=7^{\circ}$ (tool E) results in lower cutting and thrust forces. As smaller cutting and thrust forces come along with a reduced elastic spring back of the CFRP material, tools with a smaller initial clearance angle are characterised by a 
shorter arc length. This correlation can be reproduced with the wear model as seen by the comparison of Figs. 11d, 15d, and 16d. Because of a longer arc length and higher process forces, the amount of worn tool material per cutting length is the largest for the tool $\mathrm{E}(10 / 7)$, followed by the tools I (10/21) and L (20/14). While the material loss in R1 is comparable for these tools, those in R2 and R3 are the larger, the smaller the initial clearance angle is. In order to validate the wear model's capability to reproduce the influence of the initial rake angle on tool wear, the simulation results of $\mathrm{H}(10 / 14)$ and $\mathrm{L}(20 / 14)$ in Figs. 11 and 17 have to be compared. Whereas in accordance with Sect. 6.1.1, the simulation results for the tool $\mathrm{H}(10 / 14)$ agree well with the experimental data; Fig. 17e reveals that the simulation tends to underestimate the wear rate for the relatively new wear state of tool L (20/14). Accordingly, the calculated material loss in R2 is too small, which results in a difference between the simulated and measured cutting edge profiles. This difference is reduced with increasing cutting length as the WRD is increasingly overestimated with the simulation.

\section{Conclusion and outlook}

Machining of CFRP is associated to severe mechanical wear at the cutting tool resulting in a continuously changing cutting edge geometry. Common disadvantageous changes in tool shape, e.g. an increasing cutting edge rounding and a decreasing clearance angle, reduce the tool's cutting performance significantly and are the main reasons for a short tool life and damage to the workpiece. In this paper, a novel analytical wear model is presented that allows the prediction of the tool's wear progression in orthogonal machining of UD-CFRP material depending on the initial tool geometry and the fibre cutting angle. As the wear model works in cooperation with an analytical force model which was presented in a prior publication [8], the wear-related changes of the cutting and thrust forces can be tracked as well. Currently, there is no modelling approach published that allows a comparable prediction for orthogonal machining operations which makes the proposed combined force/wear-model unique.

In order to quantify the worn tool material between two consecutive wear states, the wear vectors concept is introduced. Furthermore, the WRD is defined as the plotted magnitudes of the wear vectors along the arc length of the active micro-geometry. Based on experimental data that is published in a separate publication [20], the characteristics of the WRD are analysed depending on the initial tool geometry, the fibre cutting angle and the cutting length. Based on these findings, a "line - curve - line" approach is introduced in order to approximate and simultaneously parameterise the general shape of an arbitrary WRD.
Based on a mathematical relation between the approximated WRD and the five wear parameters via the mean contact stresses and the mean sliding velocities in the three regions R1, R2 and R3, an iterative solver is applied in order to predict the tool's wear progression. Relating to a comprehensive model validation, the following conclusions can be formulated:

- In general, the proposed wear model is capable to reproduce the tool wear progression realistically in terms of the resulting cutting edge profiles, the WRD, the worn tool material and the corresponding cutting and thrust forces.

- Characteristic wear effects in machining CFRP, e.g. the increasing cutting edge rounding in $\mathrm{R} 2$, the continuously decreasing clearance angle in R3 and the elongation of the total arc length, can be reproduced in good agreement with the experimental data.

- The model's capability to reproduce the influence of varying fibre cutting angles on tool wear is validated with the tool $\mathrm{H}(10 / 14)$ in machining $\Phi=0^{\circ}, \Phi=30^{\circ}, \Phi=60^{\circ}$ and $\Phi=90^{\circ}$. Besides occurring overshooting in R2, especially for $\Phi=90^{\circ}$, the influence of the fibre cutting angle on tool wear progression can be simulated reasonably.

- The model's capability to reproduce the influence of the initial tool geometry on tool wear is validated with the tools E (10/7), H (10/14), I (10/21) and L (20/14) in machining $\Phi=0^{\circ}$. For the tested combinations, the influence of the initial rake and clearance angles on tool wear progression can be simulated reasonably.

- The proposed wear model can be used to replace the look-up table for the wear parameters $\gamma^{*}, \alpha^{*}, l_{\gamma}$ and $l_{\alpha}$ in the previously published analytical force model [8] and hence enables the prediction of cutting and thrust forces as tool wear progresses.

- By enabling the analytical prediction of the wear-related changes of the cutting edge geometry and the cutting forces, the presented force/wear-model can be used for an efficient tool geometry optimisation in terms of increasing the wear resistance.

As an outlook, ongoing research is focussing on the following topics:

- Currently, only one orthogonal machining experiment for each combination of tool geometry and fibre cutting angle is used for cutting edge interpolation and model validation. Extended experimental effort has to be provided for a detailed statistical analysis and to analyse the model robustness with respect to the variation of input parameters.

- Further optimisation is needed for the force and wear models in order to improve the prediction accuracy and to overcome the current modelling restrictions that are 
particularly found for the tool $\mathrm{H}(10 / 14)$ in machining $\Phi=90^{\circ}$ and the tool $\mathrm{L}(20 / 14)$ in machining $\Phi=0^{\circ}$. Furthermore, suitable filter methods should be integrated in order to reduce the risk for overshooting.

- Improving the force modelling approach should focus on the consideration of distributed contact forces for the chip formation process. Furthermore, additional research is needed in order to provide accurate material properties in terms of the strain rate and temperature dependencies.

- Instead of carbide tools, usually cutting tools with diamond or diamond-like coatings are used. In order to allow the applicability of the presented force/wear-model for these kind of applications, further experimental investigations are required. One important aspect that has to be considered is the influence of different wear rates for the coating and the tool substrate as soon as the protective tool coating is selectively removed.

- The presented wear modelling concept can be used for simulating the wear behaviour of self-sharpening tools. The most important requirement for self-sharpening tools is the protection of the rake face while artificially intensified flank wear resulting in a one-sided regression of the cutting edge. For this purpose, further experimental investigation is needed in order to analyse the WRD for self-sharpening tools.

Author contribution Lukas Seeholzer: conceptualization, data curation, formal analysis, investigation, methodology, project administration, resources, software, supervision, validation, visualisation, roles/ writing - original draft, writing - review and editing. Thomas Krammer: investigation, methodology, software, validation, visualisation. Parvaneh Saeedi: supervision. Konrad Wegener: funding acquisition, supervision, review and editing.

Funding Open access funding provided by Swiss Federal Institute of Technology Zurich. This research was funded by the Swiss Innovation Agency (Innosuisse Project 18309.2 PFIW-IW). Furthermore, the authors thank the companies Dixi Polytool SA, Heule Werkzeug AG, Oerlikon Surface Solutions AG and Airbus Helicopters Deutschland $\mathrm{GmbH}$ for their support.

Availability of data and material All necessary data is shown in the tables and figures within the document. The raw data can be made available upon request.

\section{Declarations}

Competing interests The authors declare no competing interests.

Open Access This article is licensed under a Creative Commons Attribution 4.0 International License, which permits use, sharing, adaptation, distribution and reproduction in any medium or format, as long as you give appropriate credit to the original author(s) and the source, provide a link to the Creative Commons licence, and indicate if changes were made. The images or other third party material in this article are included in the article's Creative Commons licence, unless indicated otherwise in a credit line to the material. If material is not included in the article's Creative Commons licence and your intended use is not permitted by statutory regulation or exceeds the permitted use, you will need to obtain permission directly from the copyright holder. To view a copy of this licence, visit http://creativecommons.org/licenses/by/4.0/.

\section{Appendix}

\section{Formulas - Voss's analytical force model [8]}

Interval I: $\Phi=0^{\circ}=180^{\circ}$

$$
\begin{aligned}
F_{I 1 \_c \_R 1}= & K_{f} A_{m b_{-} 1} \sigma_{m b}\left(1+\mu \cos \left(\gamma^{*}\right) \sin \left(\gamma^{*}\right)\right) \\
F_{I 1 \_t \_R 1}= & K_{f} A_{m b_{-} 1} \sigma_{m b} \mu\left(\cos \left(\gamma^{*}\right)\right)^{2} \\
F_{I 1 \_c \_R 2}= & K_{f} A_{m b_{-} 2.1} \sigma_{m b}\left(1+\mu \cos \left(\beta_{m b \_2.1}\right) \sin \left(\beta_{m b \_2.1}\right)\right) \\
& +\mu \frac{L_{c}{ }^{2} \pi E_{c \_\_} b}{8 R^{*}}\left(\cos \left(\beta_{p r \_2.2}\right)\right)^{2} \\
F_{I 1 \_t \_R 2}= & -K_{f} A_{m b \_2.1} \sigma_{m b} \mu\left(\cos \left(\beta_{m b \_2.1}\right)\right)^{2} \\
& +\frac{L_{c}{ }^{2} \pi E_{c \_2} b}{8 R^{*}}\left(1-\mu \cos \left(\beta_{p r \_2.2}\right) \sin \left(\beta_{p r \_2.2}\right)\right) \\
F_{I 1 \_c \_R 3}= & \mu \frac{b b_{c} E_{c_{\_} 3}}{2}\left(\cos \left(\alpha^{*}\right)\right)^{2} \\
F_{I 1 \_t \_R 3}= & \frac{b b_{c} E_{c \_3}}{2}\left(1+\mu \cos \left(\alpha^{*}\right) \sin \left(\alpha^{*}\right)\right)
\end{aligned}
$$

Interval II: $15^{\circ} \leq \Phi \leq 75^{\circ}$

$$
\begin{aligned}
F_{I 2 \_c \_R 1}= & \tau_{c} \frac{H_{1}}{\sin (\Phi)} b\left(\cos (\Phi)+\mu \cos \left(\Phi-\gamma^{*}\right) \sin \left(\gamma^{*}\right)\right) \\
F_{I 2 \_t \_R 1}= & \tau_{c} \frac{H_{1}}{\sin (\Phi)} b\left(-\sin (\Phi)+\mu \cos \left(\Phi-\gamma^{*}\right) \cos \left(\gamma^{*}\right)\right) \\
F_{I 2 \_c \_R 2}= & \bar{P}_{P 0}(\sin (\Phi)+\mu \cos (\Phi)) \\
& +\sum_{1}^{n_{B}} \bar{P}_{B i_{-} c} \frac{\Delta \omega_{i} b V_{f}}{r_{f}^{2} \pi}+\sum_{1}^{n_{P r}} \bar{P}_{P r j \_c} \frac{\Delta d_{j} b V_{f}}{r_{f}^{2} \pi}
\end{aligned}
$$

$$
\begin{aligned}
F_{I 2 \_t \_R 1}= & \bar{P}_{P 0}(\cos (\Phi)-\mu \sin (\Phi)) \\
& +\sum_{1}^{n_{B}} \bar{P}_{B i_{-} t} \frac{\Delta \omega_{i} b V_{f}}{r_{f}^{2} \pi}+\sum_{1}^{n_{P r}} \bar{P}_{P r j_{-} t} \frac{\Delta d_{j} b V_{f}}{r_{f}^{2} \pi}
\end{aligned}
$$

Interval III: $\Phi=90^{\circ}$ 


$$
\begin{aligned}
& F_{I 3 \_c \_R 1}=\bar{P}_{P b 0_{\_} c} \\
& F_{I 3 \_t \_R 1}=-\mu \cdot \bar{P}_{P b 0_{-} c} \\
& F_{I 3 \_c \_R 2}=\sum_{1}^{n_{B}} \bar{P}_{B i_{-} c} \frac{\Delta \omega_{i} \cdot b \cdot V_{f}}{r_{f}^{2} \pi}+\sum_{1}^{n_{P r}} \bar{P}_{P r j_{-} c} \frac{\Delta d_{j} \cdot b \cdot V_{f}}{r_{f}^{2} \pi}
\end{aligned}
$$$$
F_{I 3 \_t \_R 2}=\sum_{1}^{n_{B}} \bar{P}_{B i_{-} t} \frac{\Delta \omega_{i} \cdot b \cdot V_{f}}{r_{f}^{2} \pi}+\sum_{1}^{n_{P r}} \bar{P}_{P r j_{-} t} \frac{\Delta d_{j} \cdot b \cdot V_{f}}{r_{f}^{2} \pi}
$$

\section{Extended nomenclature - Voss's analytical force model [8]}

\begin{tabular}{|c|c|c|c|c|c|}
\hline $\begin{array}{l}F_{I k_{\_} c R j} \\
(j=1,2,3) \\
(k=1,2,3)\end{array}$ & Cutting force in interval $\mathrm{k}$ and interval $\mathrm{j}$ & $(\mathrm{N})$ & $\bar{P}_{P b 0 \_c}$ & $\begin{array}{l}\text { Average cutting force associated with the initial } \\
\text { fibre separation }(\mathrm{R} 1, \mathrm{I} 3)\end{array}$ & $(\mathrm{N})$ \\
\hline $\begin{array}{l}F_{l k_{-} t R j} \\
(j=1,2,3) \\
(k=1,2,3)\end{array}$ & Thrust force in interval $\mathrm{k}$ and interval $\mathrm{j}$ & $(\mathrm{N})$ & $\bar{P}_{P b 0 \_t}$ & $\begin{array}{l}\text { Average thrust force associated with the initial } \\
\text { fibre separation }(\mathrm{R} 1, \mathrm{I} 3)\end{array}$ & $(\mathrm{N})$ \\
\hline$A_{m b_{-} 1}$ & Projected area of R1 for micro-buckling & $\left(\mathrm{mm}^{2}\right)$ & $\bar{P}_{B i_{-} c}$ & $\begin{array}{l}\text { Average additional bending forces in cutting veloc- } \\
\text { ity direction (R2, I2 \& I3) }\end{array}$ & $(\mathrm{N})$ \\
\hline \multirow[t]{2}{*}{$A_{m b \_2.1}$} & $\begin{array}{l}\text { Projected area of sub-region } 2.1 \text { in R2 for micro- } \\
\text { buckling }\end{array}$ & $\left(\mathrm{mm}^{2}\right)$ & $\bar{P}_{B i_{-} t}$ & $\begin{array}{l}\text { Average additional bending forces in feed direction } \\
\text { (R2, I2 \& I3) }\end{array}$ & $(\mathrm{N})$ \\
\hline & & & $\bar{P}_{P r j_{-} c}$ & $\begin{array}{l}\text { Average additional pressing forces in cutting direc- } \\
\text { tion (R2, I } 2 \& \text { I3) }\end{array}$ & $(\mathrm{N})$ \\
\hline$E_{c_{-} 2}$ & Young's modulus of CFRP transv. to fibre axis & $(\mathrm{MPa})$ & $\bar{P}_{P r j_{-} t}$ & $\begin{array}{l}\text { Average additional pressing forces in feed direction } \\
\text { (R2, I2 \& I3) }\end{array}$ & $(\mathrm{N})$ \\
\hline$E_{c \_3}$ & Young's modulus of CFRP in R3 & $(\mathrm{MPa})$ & $R^{*}$ & $\begin{array}{l}\text { Radius approximated with least square method } \\
\text { (elliptical shape of cutting edge) }\end{array}$ & $(\mu \mathrm{m})$ \\
\hline$H_{1}$ & Height of R1 of the cutting edge in feed direction & $(\mu \mathrm{m})$ & $V_{f}$ & Volume fraction of carbon fibres in the CFRP & $(\%)$ \\
\hline$K_{f}$ & Tool-fibre contact correction factor in $\mathrm{R} 1$ & $(-)$ & $\beta_{m b \_2.1}$ & $\begin{array}{l}\text { Representative tangential angle in sub-region } 2.1 \\
\text { in R2 }\end{array}$ & $\left({ }^{\circ}\right)$ \\
\hline$L_{c}$ & $\begin{array}{l}\text { Half projected contact length of indented cylinder } \\
\text { in half-space }\end{array}$ & $(\mu \mathrm{m})$ & $\beta_{p r \_2.2}$ & $\begin{array}{l}\text { Representative tangential angle in sub-region } 2.2 \\
\text { in R2 }\end{array}$ & $\left({ }^{\circ}\right)$ \\
\hline $\mathrm{n}_{B}$ & $\begin{array}{l}\text { Total number of bending segments with multiple } \\
\text { fibre cracks (R2, I2 \& I3) }\end{array}$ & $(-)$ & $\Delta d_{j} / \Delta \omega_{i}$ & $\begin{array}{l}\text { Systematic pressing / bending displacement trans- } \\
\text { verse to fibre direction }\end{array}$ & $(\mu \mathrm{m})$ \\
\hline $\mathrm{n}_{P r}$ & $\begin{array}{l}\text { Total number of pressing segments with multiple } \\
\text { fibre cracks (I2, R2 \& I3) }\end{array}$ & $(-)$ & $\mu$ & Friction coefficient & $(-)$ \\
\hline $\bar{P}_{P 0 \_c}$ & $\begin{array}{l}\text { Average cutting force associated with the initial } \\
\text { fibre separation }(\mathrm{R} 2, \mathrm{I} 2)\end{array}$ & $(\mathrm{N})$ & $\sigma_{m b}$ & $\begin{array}{l}\text { Compression strength with respect to micro- } \\
\text { buckling }\end{array}$ & $(\mathrm{MPa}$ \\
\hline $\bar{P}_{P 0 \_t}$ & $\begin{array}{l}\text { Average thrust force associated with the initial fibre } \\
\text { separation }(\mathrm{R} 2, \mathrm{I} 2)\end{array}$ & $(\mathrm{N})$ & $\tau_{c}$ & $\begin{array}{l}\text { Ultimate shear strength of interfacial (matrix-fibre) } \\
\text { shear strain }\end{array}$ & $(\mathrm{MPa}$ \\
\hline
\end{tabular}

Table 7 Nomenclature for Voss's analytical force model 


\section{References}

1. Davim JP (2013) Machining composites materials. John Wiley \& Sons

2. Geng D, Liu Y, Shao Z, Lu Z, Cai J, Li X, Jiang X, Zhang D (2019) Delamination formation, evaluation and suppression during drilling of composite laminates: a review. Compos Struct

3. Geier N, Davim JP, Szalay T (2019) Advanced cutting tools and technologies for drilling carbon fibre reinforced polymer (CFRP) composites: a review. Compos Part A: Appl Sci Manufac 125:105552

4. Geier N, Pereszlai C (2020) Analysis of characteristics of surface roughness of machined CFRP composites. Period Polytech Mech Eng 64(1):67-80

5. Jayabal S, Natarajan U (2010) Optimization of thrust force, torque, and tool wear in drilling of coir fiber-reinforced composites using Nelder-Mead and genetic algorithm methods. Int J Adv Manuf Technol 51(1-4):371-381

6. Seeholzer L, Scheuner D, Wegener K (2020) Analytical force model for drilling out unidirectional carbon fibre reinforced polymers (CFRP). J Mater Process Technol 278

7. Che D, Saxena I, Han P, Guo P, Ehmann KF (2014) Machining of carbon fiber reinforced plastics/polymers: a literature review. J Manuf Sci Eng 136 (3):034001

8. Voss R, Seeholzer L, Kuster F, Wegener K (2018) Analytical force model for orthogonal machining of unidirectional carbon fibre reinforced polymers (CFRP) as a function of the fibre orientation. J Mater Process Technol 263:440-469

9. Dandekar CR, Shin YC (2012) Modeling of machining of composite materials: a review. Int J Mach Tools Manuf 57:102-121

10. Wan M, Li S-E, Yuan H, Zhang W-H (2019) Cutting force modelling in machining of fiber-reinforced polymer matrix composites (PMCs): a review. Compos A Appl Sci Manuf 117:34-55

11. Bhatnagar N, Ramakrishnan N, Naik NK, Komanduri R (1995) On the machining of fiber reinforced plastic (FRP) composite laminates. Int J Mach Tools Manuf 35(5):701-716

12. Takeyama H, Iijima N (1988) Machinability of glassfiber reinforced plastics and application of ultrasonic machining. CIRP Ann 37(1):93-96

13. Merchant ME (1945) Mechanics of the metal cutting process. I. Orthogonal cutting and a type 2 chip. J Appl Phys 16(5):267-275

14. Zhang LC, Zhang HJ, Wang XM (2001) A force prediction model for cutting unidirectional fibre-reinforced plastics. Mach Sci Technol 5(3):293-305. https://doi.org/10.1081/MST-100108616

15. Qi Z, Zhang K, Cheng H, Wang D, Meng Q (2015) Microscopic mechanism based force prediction in orthogonal cutting of unidirectional CFRP. Int J Adv Manuf Technol 79(5):1209-1219. https://doi.org/10.1007/s00170-015-6895-7

16. Jahromi AS, Bahr B (2010) An analytical method for predicting cutting forces in orthogonal machining of unidirectional composites. Compos Sci Technol 70(16):2290-2297. https://doi.org/10. 1016/j.compscitech.2010.09.005

17. Xu W, Zhang L (2014) On the mechanics and material removal mechanisms of vibration-assisted cutting of unidirectional fibre-reinforced polymer composites. Int J Mach Tools Manuf 80-81:1-10

18. Xu W, Zhang L (2016) Mechanics of fibre deformation and fracture in vibration-assisted cutting of unidirectional fibre-reinforced polymer composites. Int J Mach Tools Manuf 103:40-52

19. Wang X, Kwon PY, Sturtevant C, Lantrip J (2013) Tool wear of coated drills in drilling CFRPSS. J Manuf Process 15(1):127-135

20. Seeholzer L, Kneubühler F, Grossenbacher F, Wegener K (2021) Tool wear and spring back analysis in orthogonal machining unidirectional CFRP with respect to tool geometry and fibre orientation. Int J Adv Manuf Technol 115:2905-2928
21. Nguyen D, Abdullah MSB, Khawarizmi R, Kim D, Kwon P (2020) The effect of fiber orientation on tool wear in edge-trimming of carbon fiber reinforced plastics (CFRP) laminates. Wear 450

22. Maegawa S, Hayakawa S, Itoigawa F, Nakamura T (2016) Twolayer tool with hardness distribution around tool edge for reducing cutting forces in CFRP machining. Int $\mathbf{J}$ of Autom Technol 10(3):364-371

23. Caprino G, Nele L, Santo L (1996) Effect of tool wear on cutting forces in the orthogonal cutting of unidirectional glass fibrereinforced plastics. Compos A Appl Sci Manuf 27(5):409-415

24. Rummenhöller S (1996) Werkstofforientierte Prozeßauslegung für das Fräsen kohlenstoffaserverstärkter Kunststoffe. Ph.D. dissertation, Technische Hochschule Aachen

25. Henerichs M, Voss R, Kuster F, Wegener K (2015) Machining of carbon fiber reinforced plastics: Influence of tool geometry and fiber orientation on the machining forces. CIRP J Manuf Sci Technol 9:136-145

26. Ramulu M (1997) Machining and surface integrity of fibre-reinforced plastic composites. Sadhana 22(3):449-472

27. Zhang Y, Wu D, Chen K (2019) A theoretical model for predicting the CFRP drilling-countersinking thrust force of stacks. Compos Struct 209:337-348

28. Cheng H, Zhang K, Wang N, Luo B, Meng Q (2017) A novel sixstate cutting force model for drilling-countersinking machining process of CFRP-Al stacks. Int J Adv Manuf Technol 89(5):20632076. https://doi.org/10.1007/s00170-016-9236-6

29. Guo D-M, Wen Q, Gao H, Bao Y-J (2012) Prediction of the cutting forces generated in the drilling of carbon-fibre-reinforced plastic composites using a twist drill. Proc Inst Mech Eng Part B: J Eng Manuf 226(1):28-42. https://doi.org/10.1177/0954405411419128

30. Bai Y, Jia Z-Y, Fu R, Hao J-X, Wang F-J (2021) Mechanical model for predicting thrust force with tool wear effects in drilling of unidirectional CFRP. Compos Struct 262:113601

31. Luo B, Zhang K, Li Y, Cheng H, Liu SJ (2018) Modelling of thrust force for worn drill bits characterised by cutting edge radius in drilling carbon fibre-reinforced plastic/Ti-6Al-4V alloy stacks. Proc Institut Mech Eng Part B: J Eng Manuf 232(11):1960-1972

32. Henerichs M (2015) Bohrbearbeitung von CFK unter besonderer Berücksichtigung der Schneidkantenmikrogeometrie. Ph.D. dissertation, Eidgenössische Technische Hochschule (ETH) Zürich, Zürich

33. Maegawa $S$, Morikawa $Y$, Hayakawa $S$, Itoigawa $F$, Nakamura $T$ (2015) Effects of fiber orientation direction on tool-wear processes in down-milling of carbon fiber-reinforced plastic laminates. Int J Autom Technol 9(4):356-364

34. Sheikh-Ahmad JY (2009) Machining of polymer composites. Springer-Verlag

35. Seeholzer L, Voss R, Grossenbacher F, Kuster F, Wegener K (2018) Fundamental analysis of the cutting edge micro-geometry in orthogonal machining of unidirectional Carbon Fibre Reinforced Plastics (CFRP). Procedia CIRP 77:379-382

36. Seeholzer LK F Tool wear and spring back analysis in orthogonal machining unidirectional CFRP with respect to tool geometry and fibre orientation. https://link.springer.com/article/10.1007/ s00170-021-07222-z

37. Usui E, Shirakashi T, Kitagawa T (1984) Analytical prediction of cutting tool wear. Wear 100(1-3):129-151

38. Li H, Qin X, He G, Jin Y, Sun D, Price M (2016) Investigation of chip formation and fracture toughness in orthogonal cutting of UD-CFRP. Int J Adv Manuf Technol 82(5-8):1079-1088

39. Hexcel-Corporation (2010) Product Data: HexPly M21. HexcelCorporation. http://www.hexcel.com/Resources/DataSheets/ Prepreg-Data-Sheets/3501-6_eu.pdf. Accessed 8 Mar 2021 
40. Naik N, Kumar RS (1999) Compressive strength of unidirectional composites: Evaluation and comparison of prediction models. Compos Struct 46(3):299-308

41. Voss R, Henerichs M, Walch C, Kuster F, Wegener K (2016) Friction analysis between carbon fibre reinforced plastic and diamond coating with cutting process tribometer. In: International
Conference on Precision Engineering, Hamamatsu, Japan, The Japan Society for Precision Engineering

Publisher's Note Springer Nature remains neutral with regard to jurisdictional claims in published maps and institutional affiliations. 